\title{
An Ontology-based Analysis of the Industry Foundation Class Schema for Building Information Model Exchanges
}

\author{
Manu Venugopal ${ }^{1}$, Charles M. Eastman ${ }^{2}$, Jochen Teizer ${ }^{3, *}$ \\ ${ }^{1}$ Autodesk Inc., 111 McInnis Pkwy, San Rafael, CA 94903, PH +1-646-455-9685, e-mail: \\ manu.venugopal@autodesk.com \\ ${ }^{2}$ College of Computing and Architecture, Georgia Institute of Technology, 247 Fourth Street \\ N.W., Atlanta, GA 30332-0155, PH +1-404-894-3477, FAX +1-404-894-2278, e-mail: \\ charles.eastman@coa.gatech.edu \\ ${ }^{3}$ RAPIDS Construction Safety and Technology Laboratory, Germany, PH +49-157-5476-9592, e- \\ mail: jochen@teizer.com (*corresponding author)
}

\begin{abstract}
Robust knowledge sharing frameworks between different stakeholders in a building project is of high priority. Industry Foundation Classes (IFC) provides a rich schema for interoperability through objectbased transactions. However, IFC lacks semantic clarity in mapping entities and relationships, resulting in multiple definitions to map the same information between different federated models. The objective of this research is to examine IFC from a perspective of an ontological framework, which can make the IFC definitions more formal, consistent and unambiguous. Different methods of ontological approaches to engineering knowledge are reviewed. Various issues such as the need for a logical framework, the current semantic approaches in the AEC/FM industry, and advantages of building an ontology structure are addressed. A comparative study of the ontology and segments of the existing IFC schema definition are performed. This exercise reveals the ambiguous nature of current IFC definitions and proposes reforms such that data exchanges would be more semantically robust. An ontology would structure the overall interoperability of BIM tools by providing a formal and consistent taxonomy and classification structure for extending IFC and for defining subsets as model view definitions (MVD).
\end{abstract}

Keywords: Building Information Modeling (BIM), Product or Process Modeling, Model View Definitions (MVD), Industry Foundation Class (IFC), Ontology, Semantic Exchange Modules (SEM)

\section{Introduction}

As the world of Building Information Modeling (BIM) expands, processes including ubiquitous computing make information available to the right person at the right time. Servers, such as those on the cloud, need to evolve toward platform agnostic software for the AEC/FM industry. The importance of robust knowledge sharing frameworks between different stakeholders is of highest priority in many application scenarios [1]. Industry Foundation Classes (IFC) provide a rich, redundant but ambiguous schema for interoperability. An important issue is that IFC lacks semantic clarity in mapping entities and relationships, resulting in the potential for multiple data structures to represent the same information. This is necessary because building models require different semantics for different workflows over a project's lifecycle. The objective of the research reported here is to define an ontological framework which makes the IFC definitions more formal and consistent such that data provide an improved basis of information exchanges than what exists today. A formal specification of IFC entities to create a taxonomy of the classes is outlined. Various issues such as the need for a logical framework, the current semantic approaches in the AEC/FM industry, and advantages of building an ontology structure are addressed. A comparative study of the ontology and the existing IFC schema definition is performed. It illustrates the ambiguous nature of IFC definitions and proposes reforms that will lead to future data exchanges being more robust. This research is expected to impact the overall interoperability of BIM tools by providing a formal and consistent taxonomy and classification structures for creating new model view definitions (MVD)s,. It provides the mechanism for applications making data sharing across cloud or federated servers simpler with limited rework. 
Interoperability is defined as the ability of diverse systems and organizations to work together or interoperate. The problem of interoperability in the BIM landscape is well documented [1] and is estimated to be costing the industry more than 15 billion US dollars every year [2]. Eight in ten users of BIM software tools in the US consider interoperability or lack of it between software applications to be the limiting factor in achieving the full potential of BIM [3]. The Industry Foundation Class (IFC) schema is accepted as the industry standard for interoperability [4-5] and is an official international standard (ISO/IS 16739) [6]. The review here of IFC pertains to Release $2 \times 3$ only. Recent changes made in Release 4 may alleviate some issues raised.

The core concepts for IFC were developed in the late 1980s and early 1990s, before the general recognition that multiple federated models are necessary in building design and construction and before the recognition of cloud computing and the potential of background automated file exchanges. There is no mechanism to edit the subset of a model and reliably integrate the edited parts beyond geometrical considerations; systems and groups are assumed to all reside is a single runtime accessible structure.

Model exchanges based on IFC are still file-based and error-prone [7]. Errors still occur when both translators involved in exchange are IFC compliant; exported data needed for a targeted exchange but semantically not understood by the importing application. Hence, object schemas such as IFC are necessary but not sufficient for achieving robust data exchanges. Current model view development methodologies leave room for different interpretations based on end-user requirements. The results are that one exchange may use one set of information structures to define a semantic condition, but a separately developed one may structure data in an incompatible manner. Lacking is a formal framework for deriving semantically driven views from models generated from different applications. Moreover, the granularity and atomicity with which such model views are defined is not consistent across the industry [8]. This adds to the overhead for software developers and hinders IFC based implementations [9]. Hence, there needs to be a better way to consistently specify IFC implementations based on exchange requirements. In order for that to happen, additional levels of specificity are required to define model exchange requirements and model views in a formal, consistent, modular, and reusable manner. This research aims to improve the data sharing for the AEC/FM domain by providing a formal definition of IFC entities, relations, attributes, and methods for information exchange, using an engineered ontology. Ontology in this regard can be considered as a machine-readable set of definitions that create a consistent taxonomy of classes and subclasses and relationships between them [10].

The gaps in interoperability research are introduced and explained in the background section. The need for formal methods is summarized, followed by an introduction and survey of engineering ontologies and their uses. Some parallels between the research objectives of other knowledge sharing paradigms and product model exchange research are investigated. Research in the AEC/FM industry focusing on this area is discussed in the summary of this section. The research methodology and the framework are presented in the next section. The rationale for developing an ontological foundation for IFC is provided. A comparative study of the ontology structure developed in this research and the current IFC schema structure are performed to reveal ambiguous definitions. An example is developed in the form of two application areas in cast-in-place concrete and precast/pre-stressed concrete industry using a model view developer plugin. The conclusion discusses the impact of this research in developing robust and consistent model views and also future applications such as easing the testing and validation requirements for IFC implementations.

\section{Background}

\subsection{BIM Interoperability in the Cloud Realm}

The AEC/FM industry is following the general trend in the software industry and is moving towards cloud-based, scalable, and ubiquitous architectures to support model creation, data sharing, and 
information consumption. Existing interoperability standards and software methodologies, however, slow down this industry trend and rather creates information silos than well-connected systems.

The National BIM Standard ${ }^{\mathrm{TM}}$ initiative (NBIMS) [11] proposes facilitating information exchanges through model view definitions (MVD) [12-14]. A model view is defined as a subset of the building product model schema that provides a complete representation of the information concepts needed for a particular use-case in an AEC workflow [8]. For example, during a project lifecycle, information is generated by architects, engineers, detailers, estimators, construction managers, facility managers, and numerous more project stakeholders. Information handling can grow to unmanageable proportions. There is a need to extract the minimum information necessary to support a particular use-case such as coordination, quantity-takeoff, model based scheduling or commissioning and handover, or maintenance. This minimum sub-set of information that needs to be extracted from a client workstation or server is defined as a model view. The model view approach defines the appropriate information entities from a schema for particular exchange requirements (ER) and the rules regarding their instance population. This step is performed by documenting them in an information delivery manual (IDM), and mapping these to the IFC schema definitions defined in a MVD.

The work done on the precast national BIM standard [15], which is one of the early NBIMS, has given insights into the advantages of the MVD approach. This has enabled identification of areas that require attention, leading to the research presented in this paper. Some of the results are being implemented in improving the precast NBIMS, supported by the Precast/Pre-stressed Concrete Institute (PCI), and also in the current development of national BIM standards for cast-in place concrete [16] and the steel industry [17]. They are supported by the American Concrete Institute (ACI) and the American Institute of Steel Construction (AISC), respectively. Interoperability enhancements require common understanding of industry processes and the information required for and resulting from executing these processes. Two sets of semantics are at the core of any model view specification [8], namely: (i) the user/application functional semantics defining the information that must be exchanged, and (ii) the representational semantics available in IFC or other data-modeling schema for representing the user intentions.

IFC is highly object-oriented supporting inheritance, polymorphism, and extensibility. Objects are represented as functional classes connected through objectified relationships and attributes. IFC supports multiple kinds of representations and open-ended value properties. As the coverage of the schema has grown, it has become difficult to keep the extensions and modifications semantically consistent. Any person defining models in IFC (or other schema) asks and resolves the following example types of questions. How does one represent in IFC: type-instance relations, shape families (may be different than type instance), patterns of layout, such as rebar, tiles, brick (at the level of detail needed for fabrication), based on forms of aggregation, embedded relations such as for connections and embedded elements, nonoverlapping but tightly packed relations between objects, such as precast concrete pieces and slab assemblies, relations between objects to reflect different semantics: connection, association, assembly, alternative model views for the same object, for fabrication, as installed (with deformations), and analytic models, and others.

Exchange models are found to be repetitive and these repetitive specifications, which form the building blocks of a MVD, were termed IFC Concepts [18]. The idea of IFC Concepts was introduced as a means of modularizing MVD development and also for improving re-usability. However, the two public support facilities available for model views assume varying levels of granularity and atomicity of the building blocks for an MVD [19-20].

Figures 1(a) and 1(b) show a subset of IFC concepts as defined by the IFC solutions factory and the GTDS projects, respectively. Concepts in the areas of engineering and design are particular, in the sense that they define a mixture of partial specifications of reality, the expected function and behavior of that 
reality, and the reality of physical systems. Concepts regarding the different levels of building realization are needed to distinguish between conceptual designs and fabrication details within a construction domain. IFC provides a schema made up of its own set of concepts to define instances of specifications of both designs and real buildings. Buildings are described in terms that vary in their generality and like other taxonomies of engineering and design, with varied levels of realization. Buildings are made up of many different systems that each have their own concepts, as well as shared ones, that are authored in different applications. This implies that there is more than one way of representing the information to be exchanged or extracted to or from a federated model server.

A first step would be to make the background meaning about the IFC concepts that are currently implicit to be made more formal and explicit. Even though IFC Concepts are being used by most of the NBIMS model view efforts (there are published over 60 top level concepts and more than 1080 bottom level binding concepts), there has been no formal specification of what an IFC concept is and how they should be structured, as required for re-use. IFC Solutions Factory [20] is a shared repository for model view development work available around the world. Most of these efforts are generating concepts with IFC bindings in an ad-hoc manner suitable to their specific application requirement, with limited re-use across such efforts.

The General Testing and Documentation Server (GTDS) [19], is an online database and test center supporting the official IFC certification procedure. GTDS aims to automate the testing of IFC files based on validation rules that formalize the constraints imposed by the model view definition. Export test instructions, import calibration test files, validation rules, and more are under development to automate the certification workflow based on IFC Concepts. For example, the GTDS testing facility groups the concepts at the building element level (macroscopic approach) as seen in Figure 1a. Such a hierarchical structure coupled with manual rule testing for each entity allows verifying the correctness of test files from each entity level. However, this approach has several limitations as well, such that testing cannot be complete as there is no means to verify the semantic correctness of relationships and patterns of entities. On the other hand, the IFC solutions factory approaches concepts from a microscopic level, whereby each and every IFC entity is defined as a concept (see Figure 1a).

Figure 1: Concept structure for the IFC schema supported by two industry mechanisms: (a) IFC solutions factory hierarchy of concepts [20] and (b) Concept structure for certification testing on GTDS platform [19]

For example, even a globally unique id, which is an attribute of an element, is defined as a concept. This approach allows performing unit testing of concepts to make sure they are correctly defined and also reusable to a limited extent. However, testing the composability of a complex model exchange using such microscopic concepts is not feasible at this stage.

This research proposes a clear and formal definition of IFC concepts, based on an ontology structure, outlined here, making them reusable and providing a taxonomical library for defining and adding model view definitions. The following section illustrates some cases of the ambiguous nature of the IFC schema definition.

\subsection{IFC Semantics}

IFC is strongly object-oriented and uses inheritance as a fundamental structure. Thus, inconsistencies are not apparent at the level of specification. See for example Figure 12. What are the distinctions between IfcElementComponent, IfcBuildingElementPart and IfcDiscreteAccessory? With a well-defined highlevel abstract ontology, these distinctions can be defined [21]. However, there are in the current structure with alternative ways to represent the same thing- because the rules for discriminating them are not defined. These redundancies raise questions about which of the ways should be used to represent a 
particular condition. Defining the semantics of each of the objects, relations, and other constructs used in IFC according to their often implicit meanings is needed.

Semantics potentially become more explicit during the development of model views. For example, the available geometry for the connection constraint may be further restricted to only allow straight segments by applying IfcPolyline only.. Concepts identify issues about the proper use of redundant methods to specify a concept, or in the lack of specification of an entity. For example, objectified relations in IFC cause potential problems. They are not first class objects (not dependent for its existence on other data) because they are applied contextually to link different objects, usually pair-wise. Their links to the RelatedObject and RelatingObject are not optional. This means that a relation is dependent upon the a priori existence of the relation and related objects, and must be automatically deleted if either of them is deleted. Because of its structure and this behavior, it should not be considered a first class object, even though Relations are children of IfcRoot, which is the base object for first class objects.

There are other ambiguous relationship entities. Assemblies are defined with IfcRelAggregate relation. An aggregation is a general collection of entities of varied type. Currently, no distinction is made between objects assembled so they cannot overlap (steel assemblies) and those that can overlap (cast-in-place concrete elements). This makes the general computation of properties of an assembly often impossible or erroneous. For example, in some cases the volume of an assembly is the sum of its parts volume, while in other cases it is not.

The notion of human cognition versus machine readability is another important aspect to be considered for resolving interoperability. The human mind is able to think at different levels, regarding varied instances, whereas machines need clear definition of the semantics. For example, it has been shown that reinforcing elements can be aggregated in at least three different ways and each case can be used as a type [22]. IFC allows various ways of defining object instances and object types. Types are defined at multiple levels of abstraction. There is an urgent need to review the types of relationship objects, to examine their use and dependencies.

\subsection{Summary}

IFC lacks the formal semantics to relate the issues identified above such as typing, classification and relationships, to reasoning mechanisms. There is no firm class or property hierarchy (except for a building-oriented spatial hierarchy) as most classes and properties are subclasses of the top-level entity that is IfcRoot. Further, many objectified relationships take IfcRoot as their domain or range. Some of the implicit semantics are in the IFC documentation whereas some semantics are left to the users or future work. To overcome this situation, the level of formal definitions and foundations, what can be referenced as commitment in IFC will need to be raised and we recommend a formal definition for IFC extensions, by identifying the underlying concepts used to define the meaning of IFC entities, relations, attributes and rules, as these are the basis for the IFC structure. Garrett et al. [23-25] utilizes text analysis algorithms to solve classification issues, whereas semi-automated approaches for the integration of project documents to model objects are also available [26]. The need for advancing formal methods for data collection, data mining, data analysis, and project planning and control are well documented [27]. These issues require full understanding by the relevant users, and their unambiguous mapping to IFC for effective exchanges. Several ambiguities have been initially defined, but have not been checked by the IFC Implementation Support Group (ISG). How to use these IFC entities, how to properly use them in concepts, are among the challenges one has in writing IFC models for particular use, especially for model views.

This research intends to define and document these ambiguities and provide guidelines for future users in the form of one or more ontologies for IFC. The current status of model exchanges using IFC is summarized as follows [8]: 
1. IFC is rich and redundant offering multiple ways to define objects and relationships (to reflect user intention).

2. The development of an information delivery manual (IDM) is based on industry knowledge and human expertise.

3. The translation from IDM to MVD is largely manual and requires significant trial-and-error.

4. The base concepts for exchange (IFC Concepts) are not strictly defined and as a result have multiple versions.

5. The IDM/MVD is not based on logic foundations, and hence, thel application of machine-based reasoning mechanisms is not possible.

6. The required level of detail for most model exchanges is not specified.

The following section explores the different mechanisms to formally represent information from the area of computer science and knowledge representation and sheds light on the suitability of such approaches to the issues faced in AEC/FM.

\section{Knowledge Representation Mechanisms 3.1. Formal Methods}

Knowledge representation is the field of artificial intelligence that focuses on the design of formalisms that are both epistemologically and computationally adequate for expressing knowledge about a particular domain [28]. Description logics (DL) and frame-based systems are tools that enable the researchers to represent knowledge in a structured way that support automated reasoning. A DL model consists of a domain and an interpretation function. The domain is the set of objects and the interpretation function is a mapping from individual, class, and property names to elements of the domain, subsets of the domain and binary relations on the domain [28]. The key feature of DL's is that they are formal languages with welldefined semantics. Importance is given to formally defined relationships (i.e., subsumption, equivalence) between the objects. DL help information to be shared without any misinterpretation of the terminology and their meaning. Frames paradigm can be seen as an alternative to DL's with the goal of making formal representations easier to read and understand. Frame based languages represent each class as a frame, where information about the hierarchical structure of the class, name, and other properties (slots) are defined.

A class frame is semantically equivalent to a DL axiom. The need for such formal representation of exchange requirements was observed in various ways model views can be generated using IFC for the same exchange [9]. An important advantage of formalizing the terminology is that automated reasoning of business rules to check consistency of classes and model view definitions becomes possible. However, there are trade-offs between providing expressivity and formal tractability. It is a computational balance, which is important in the case of rich schemas like IFC. It is important that the constructors and axioms developed can support practical decision procedures. Boolean operators such as intersection, union, and complement are widely used for geometry. Property hierarchy is an important aspect of IFC, which provides transitive properties as well as restrictions on properties. Inverse properties are widely used in IFC, which also provides enumerations for classes.

\subsection{Engineering Ontology}

Ontology is a formal representation of an abstracted view of a domain that describes the objects, concepts and relationships between them that holds in that domain for a stated purpose [10]. There are different classifications of ontologies, based on parameters such as level of granularity, their use and types of relationships [29-32]. Formal ways to deal with ideas such as description, attribute, concept, role, inheritance and instantiation were introduced paving the way for future ontology languages [33]. Some of the language formats developed over the past two decades are reviewed here, with the idea of representing EXPRESS/IFC based data schema in one of them. 
The Knowledge Interchange Format (KIF) is an formal approach used for knowledge exchange among computer programs that are different in nature [34]. The semantics of KIF are based on the correlation between the terms and sentences of the language and the conceptualization of the world in terms of objects, functions and relations. KIF uses declarative semantics for representing the meaning of expressions using first order predicate calculus and reasoning rules. This is a very early approach and lacks in its inability to transmit declarative information between large systems. Moreover, a declarative database created for IFC should be more than just a collection of first-order sentences. KIF has been the foundation for much recent work such as ontolingua server [35], which allows users to develop high-level ontologies by using predefined ontological descriptions.

F-Logic is another approach, where well-defined semantics of logics are integrated with frame-based languages to provide formal semantics and resolution-based proof procedures. This was developed particularly as a database logic language comprising the object-oriented features such as object identity, complex objects, inheritance, methods, and rules [36]. Inference engines for F-Logic are efficient and recent approaches to represent ontologies in F-Logic have been successful [37].

Structure-function-behavior (SFB or SBF or FBS) is another knowledge representation paradigm for engineered products where information is grouped according to the material and geometry of the product's structure, the model's function, and behavior in order to construct a clear, consistent, computable, and widely useful model [38]. This is also known as teleological modeling of a system, which is a representation that specifies both functions of the system and the causal processes that result in the system functions, at multiple abstraction levels. Computational research on engineering design and problem solving using teleological modeling is reported in [39-41]. At the highest level, an SBF specification looks as follows [42]:

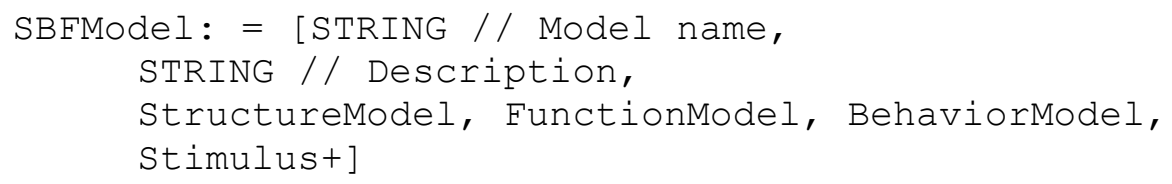

A StructureModel consists of the elements and the connections among the elements. A BehaviorModel is nothing but a set of states of the model, defining relevant performances, and related transitions between them (usually abstracted from computational methods that derive the states from structure parameters). The FunctionModel describes the purpose or goal of the element, which is achieved through its behavior [42]. The external effects on the behavior of a model are represented through Stimuli. SBF models of complex systems enable computer programs to draw inferences about the systems. SBF provides benefits to knowledge representation by means of precise vocabulary, consistent usage, and tool building methods or model reasoning regarding the behavior and structures associated with various functions.

\subsection{Semantic Web Technologies}

The scope and potential of BIM is ever-increasing as a result of new IT-enabled approaches to facilitate design integrity, virtual prototyping, simulations, distributed access, retrieval and maintenance of project data between multiple disciplines [43-44]. There are parallel approaches to introduce semantics into building information modeling, by means of using web standard technologies (W3C) and techniques [21, 45-46]. They include the use of formal methods such as Resource Description Framework (RDF) schemas, functional specifications and ontologies. Semantic web is an example of inter-linked data available in a standard format, reachable and manageable by automated tools. Similar sets of issues are faced by the semantic web development effort as are found in IFC interoperability. Hypertext markup language (HTML), which is popular as the web language, is designed to provide descriptions of documents and inform a browser on how to render the contents, for example, font size and color. While not recommended, HTML provides limited scope to express semantics using meta tags. 
Extensible markup language (XML) was developed to separate the markup of web content from presentation, thereby streamlining task specific and domain specific data. This is not a semantic language and relies on document type definition (DTD) to enforce constraints on which tags to use and how to use them. XML is widely used on the web and has been successfully implemented as an exchange format for EXPRESS data. Representing model views in XML, called mvdXML, provides a computer readable format, however it lacks formulating axioms and cannot be considered an ontology. The semantics expressed using markups are visible to computers only through the use of external agreements and meaning of annotations. Use of an ontology to specify the meaning of such annotations has been shown in research.

BuildingSMART has recognized mvdXML as the standard for representing model views [47]. Providing a formal ontological structure from which mvdXMLs can be generated would be beneficial to the industry. The complete XML schema file can be downloaded [48]. RDF is the standard developed for describing any web resource in a machine understandable way while exchanging information. RDF strives to add a formal definition to the web by providing a data model and syntax conventions based on the object-attribute-value, commonly known as the RDF triplets, as shown below:

<rdf:object, rdf:attribute, rdf:value>

For example, a building that has a unique ID, name, owner history, and placement can be represented as follows in RDF. The owner history and placement are referring to another instance in the file whereas ID and name is a value. This provides a machine-readable format from which graphs can be generated by applications reading this data.

The main advantage of RDF over basic XML is that RDF can define a basic set of terms with specifiable meanings in the form of a schema or layer on top, called RDFS. Using a schema with entities such as rdfs:class, rdfs:subclass, rdfs:property, rdfs:domain, and rdfs:range we can model a hierarchy of classes and properties with domains and restrictions. In RDF, the schema is separate from the data and any model can reference and share the schema (like IFC). Attempts were made to utilize the large user base for unified modeling language (UML) [49] and develop ontologies based on UML. However, a major drawback of UML for developing an ontology is that it lacks the formal specifications that form the backbone of ontologies. XML Schema definition language, on the other hand, is a direct machinereadable mapping from IFC to XML schema, facilitating automated mapping [48].

Web ontology language (OWL) is the formal ontology language developed for the semantic web. There are two alternative ways to provide semantics in OWL, direct semantics or RDF-based semantics. Semantics are necessary for reasoning tools, to check consistency, subsumption relationships and querying. OWL data types are based on the XML schema definition language (XSD). OWL/XML, which is an XML serialization, provides users with a variety of options to process information using XML tools. In this research, it has been used to formulate a model view in the form of mvdXML. With its highly intuitive, compact syntax and well-defined formal semantics, this ontology is able to represent knowledge and define the relationship between terms allowing applications to interpret their meaning in a flexible and unambiguous manner and enable reasoning capabilities. The snippet below shows an example of semantic web ontology language (OWL/XML) representation for building elements.

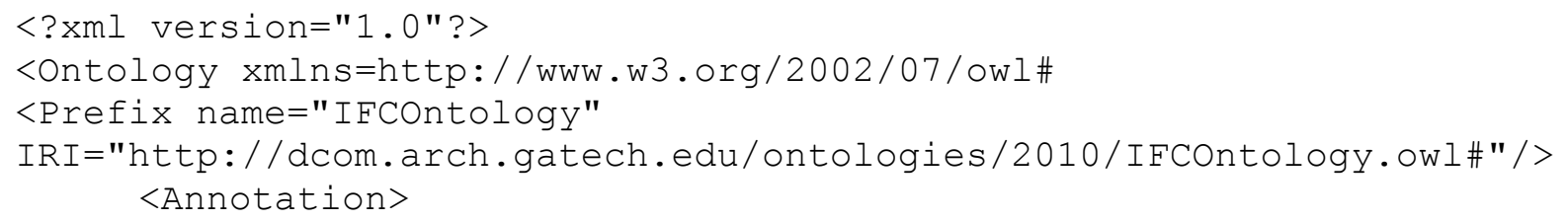




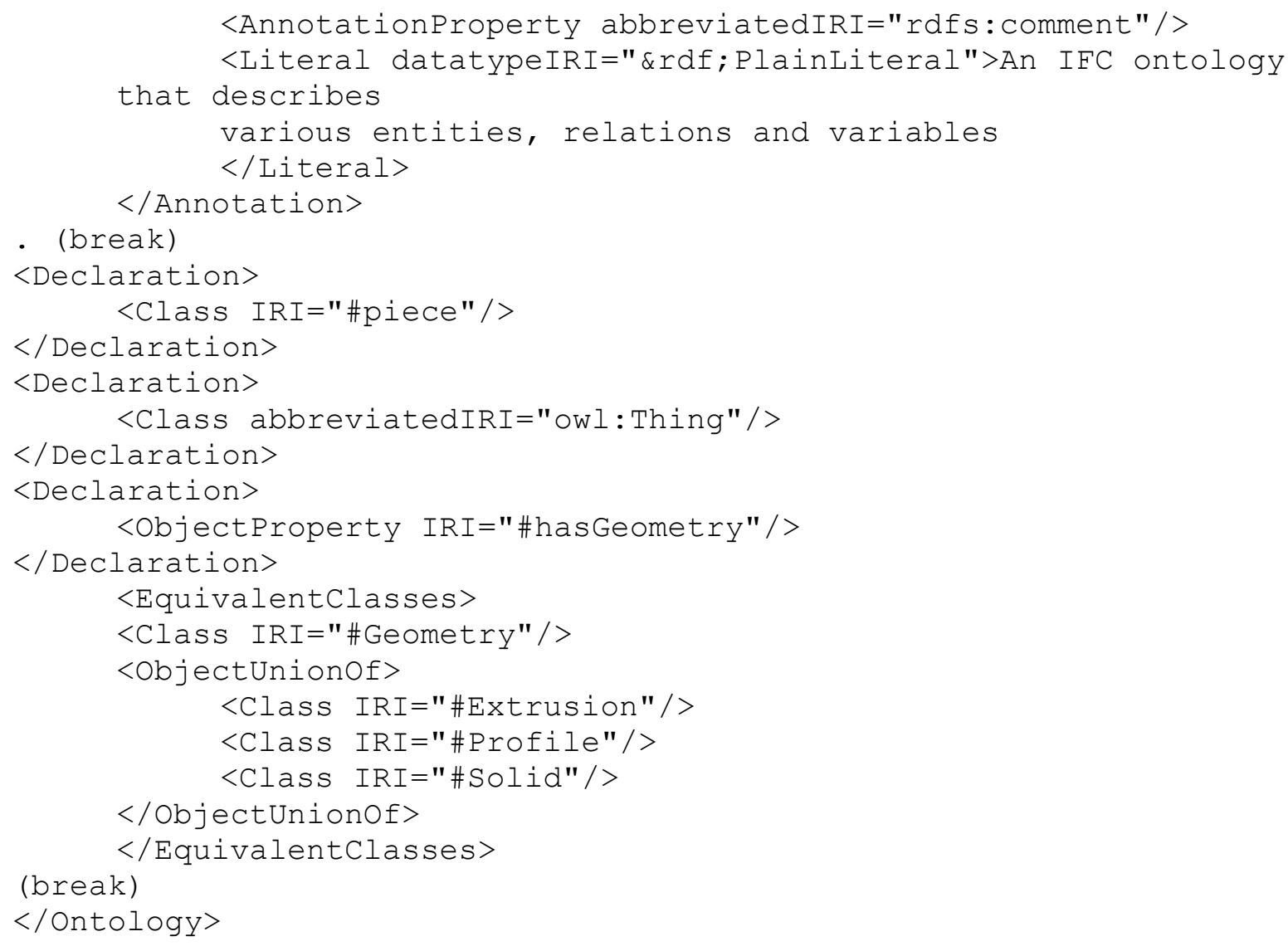

The snippet is based on the existing IFC schema definitions. Please note, the objective of this research is to define ontology definitions independent of the IFC schema and then perform a comparative study to understand the ambiguities present in the IFC schema definitions.

Defining ontologies by implementing them directly in ontology languages can be time consuming. We explored the use of tools currently available for ontology development and decided to use Protégé. The literature provides information about many other editors and tool kits, such as, The OntoLingua Server, WebOnto, OilEd, WebOde, OntoEdit, KAON, etc. [50-52]. The concept behind Protégé as defined by its author's states knowledge acquisition proceeds in well-defined stages and that knowledge acquired in one stage could be used to generate and customize knowledge-acquisition tools for subsequent stages [53]. Evolution of Protégé is explained in [54]. Some of the main features of this platform:

1. Based on frames/OWL and first order logic,

2. Modeling component classes, slots, facets and instances,

3. Supports rule-based classifications, and

4. Classes can be concrete or abstract.

Rule-based checking approaches follow a semi-automatic mechanism of pre-defining some of the semantic based concepts using a mark-up methodology and development of computable rules [55]. However, at this point of time, many of these approaches are limited to custom domains and specific applications and are not applicable as a general and industry-wide solution for AEC/FM. At the same time, the issue of semantic robustness of model exchanges using IFC needs to be seriously considered for advancing interoperability within the AEC/FM industry. The MVD development process needs to be transitioned from the current ad hoc manner to a rigorous framework and methodology. 
The authors believe that the current ad hoc methods are not scalable to more than a few MVDs, each defined laboriously. The semantic meaning of IFC Concepts needs to be defined in a rigorous and formal manner with strict guidelines. A logical framework on the basis of well-defined and unit tested IFC concepts, thereby following a modular approach, should be the direction for creating MVDs in a standardized and re-usable manner across domains. The next section explains how engineering ontologies are used in this research to arrive at a formal approach for developing model views.

\section{Framework for interoperability in AEC/FM}

With regard to coverage, the IFC data model has received more than three decades of development/refinement. It currently represents subtle knowledge in a wide range of construction domains. It will never be complete, given the open domain of a large world-wide industry with many paths of innovation. While some of its features are laudable, its foundations have weaknesses that are cumbersome, time consuming and ambiguous for users. It relies on a process that involves a wide range of expertise at both the domain and IT levels. These weaknesses will likely lead to growing problems as it expands into ever wider utilization. This paper identifies some of those weaknesses and outlines a path for addressing the weaknesses.

\subsection{Research Questions}

This research is meant to define a framework that re-defines the way interoperability is approached in the AEC/FM industry. It addresses the following question:

Can a well-defined method for semantic classification and representation of IFC entities be developed? The practical objective of this goal is to determine a data model that can represent the range of semantic structures needed to address building model semantics.

The longer term objective is to adapt IFC schema to support easy definition of model views that are consistent, testable, extensible and re-usable across various domains in the AEC/FM industry. This aspect addresses the procedures needed to support interoperability, regarding such issues as knowledge capture and requirements definition, efficient support for translation mappings between a group of heterogeneous platforms, and validation testing. We consider the above question to be prerequisite to these more extensive set of issues.

The proposed research framework is shown in Figure 2 and explained in the following sections.

Figure 2: Framework for an ontology based approach for interoperability in AEC/FM.

\subsection{Research Methodology}

The first step in the framework is an in-depth analysis of the IFC product modeling schema to identify the explicit semantic classes needed to distinguish the different meanings and related issues in model exchanges. It sets out to identify the semantic distinctions upon which the current version of IFC is defined. Such an exercise forms the backbone of the ontological definitions to be developed. The results provided by the semantic analysis are relatively informal in nature. However, the analysis intention is to identify the information classes, criteria, and properties that are used to define the high-level abstract semantics of the ontology. IFC already groups semantic classes of entities at a high level within IFC: BuildingElements, BuildingComponents, DistributionElement, ControlElements, ProcessElements, Actors, Groups, and Resources. Each IFC class has a description characterizing the criteria for the class. These should be articulated and expanded for the various subclasses of the high level classes addressed. The goal is to identify a compact set of such information that is sufficient to semantically distinguish the objects and relations encountered in IFC, for the domains of IFC addressed, as shown in Figure 2, the outputs of this stage are textual descriptions of the semantic rules and classifications that are embedded in 
IFC schema, but not necessarily articulated through constraints. The detailed analysis of the IFC schema, some initial results, and recommendations can be found in [8].

The second phase involves the development of an ontology-based definition for IFC based on the classifications defined in the first phase. This phase includes the preparation of a knowledge base, setting up the modeling criteria, and assumptions. The development on the ontology structure is a key aspect in knowledge representation and sharing, as it provides a rigorous bridge between human and computer understanding. The ontological definitions are developed by adding detail where necessary, incorporating formalisms wherever helpful, and generally enhancing the consistency and modularization of IFC entities, attributes, and relations. This is the major focus of this paper. For example, what IFC entity can be used to represent openings in a slab? Is there a way to differentiate openings that are through the element such as for doors and windows and recesses and niches which are meant as pockets for embeds or other connections? What sort of relationship is valid between the parent element and the opening and also to the element that goes into the opening? Are there any additional rules that need to be applied for precast specific elements or for steel fabrication?

A clear ontological classification of IFC entities allows for unambiguous representational semantics. In the ontological framework, domain knowledge is divided into smaller pieces called engineering ontologies, that addresses, for example, geometry, geometric distortions (camber, deflection, warping), components, connections, properties, placement hierarchies, relations,. The application ontology can be constructed by the integration of these smaller pieces. The precast piece application ontology is developed using Protégé and is available in OWL form. However, there is limited use for the ontology in the form of OWL representation, and an additional layer of mapping is provided for implementation. The semantic classes provide a formal but human readable set of classes supporting modules, potentially supporting composition as modules to define new semantics to support exchanges based on different semantics.

Semantic Exchange Modules (SEM) provide the additional implementation layer in the form of objectoriented libraries developed on top of the ontology. The ontology layer provides the abstract structure of the schema, whereas, the object-oriented layer provides the actual implementation structure in the form of data and code. Moreover, the physical representation of the data structures and methods are important and described in the SEM layer [56]. A model view defined on the basis of ontology structure and presented in the mvdXML format is provided as the input for the SEM mapping layer. Algorithms and methods are being developed to map this SEM layer to IFC and also to native data structures of multiple BIM software. These implementation level details form the basis for the third phase in the framework, but are out of scope for this paper.

Validation and verification, which form the final phase, are concerned with ensuring semantic correctness of the defined product. For the purpose of the research in this paper, these questions can be translated to verification focuses on the correctness of the mvdXML structure developed in relation to the ontology and validation equates to checking whether the mvdXML development-based ontologies actually cover the semantic needs identified. These issues are addressed in the form of a proof of concept implementation called model view developer plugin in C\# language. Some of the applications of such a framework include, but are not limited to, developing modular MVDs, satisfying model exchanges for certification and testing, and querying of model data. The following section introduces the research methodology followed for the development of the ontology structure.

\section{Ontology Building}

The main focus of this phase of the research is in formalizing the exchange semantics using an ontological backbone and mapping them to IFC entities, relations, attributes and functions into semantically defined modules. The objective of the ontology development is to remove ambiguities 
associated with differing viewpoints [57] and formalize IFC definitions for a robust model exchange solution as shown in Figure 3.

To illustrate this, let us consider a use case involving information exchange between a precaster and the architect/engineer (Figure 3(a)). A precast piece such as a floor slab can be represented early-on as a monolithic slab entity in the structural design model (Figure 3(b)) developed by the architect/engineer, whereas the fabrication model developed by precaster will include the high level details of discretized hollow core slabs for the entire span of the floor, topping, along with the connection details, finishes, joints, embeds, reinforcing, tensioning cable layout, lifting hooks, and more (Figure 3c). This is an instance of the Level of Development issues being proposed by AGC [66]. The corresponding IFC entities and relationships will also be different as shown in Figures 3, 4, and 5. The structure of a model view for the exchange of product model data between various BIM application tools depends on the extent to which building function, engineering, fabrication and production semantics will be embedded in the exchange model, first at the source building model by the modeler and the capability of any receiving application to correctly interpret and incorporate the model semantics in an articulated semantic structure. In order for the importing application to infer semantic knowledge from the exchange, the exporting application should structure the data based on an agreed upon standard. The ontology definitions provide a means to remove ambiguity regarding those different meanings in such scenarios, by using constraints that define the relationships and also provide equivalences between individuals. For example, is a structural member represented in its fabrication shape or as installed with camber and other distortions?

Figure 3: Example of different viewpoints: (a) structural model and fabrication model representing two views of the same entity, (b) design model - monolithic slab, (c) fabrication model -parent slab detailed as individual hollow core planks with connections and reinforcing.

Figure 4: IFC modularization for mapping a monolithic precast floor slab, organized as modules: Material Association, Placement, Spatial Containment and Manifold Solid Geometry

Figure 5: IFC mapping for a floor slab with discretized hollow core precast slabs. Hollowcore and topping are both subtypes of BuildingElement, which together form a slab assembly, with placement and properties.

The section on ontology definitions, later in this paper, illustrates how constraints are used to restrict representational semantics. In terms of the modeling criteria, a corpus or body of knowledge needs to be constituted. Entities are selected from the available domain-specific documentation according to the ontology requirements. The precast NBIMS model view in general and the building components in particular are selected as the corpus in this case. Once the ontology is developed and validated, it can then be applied to other domains as well such as cast-in place concrete and steel industry. Protégé and web ontology language (OWL) are the tools that are used to represent knowledge in a structured and reasonable way and the final model view is in the form of mvdXML. The IFC data model is envisioned for different services across various domains, whether for a specific data exchange between a precast detailer and structural engineer, or between an architect and MEP contractor. The descriptions of services should be formulated according to the ontology in order to support the automation of service related tasks. These types of services can be defined as some of the uses of the ontology for the IFC data model.

Main criteria for development of an ontological structure is knowledge sharing and reuse, hence it is evident that the ontology developed will be large and complex. A divide and conquer approach [58] is adopted in developing this ontological structure. The methodology for the ontology building is shown in Figure 6. A sound base is important for building any hierarchy. This is achieved in this research by structuring the ontologies on a foundational ontology such as descriptive ontology for linguistic and 
cognitive engineering (DOLCE) [59]. This will be the most abstract layer, introducing the basic modeling concepts and generic design guidelines for the construction of actual ontologies.

Figure 6: Overview of an ontology structure for precast application ontology.

The second layer consists of super theories such as mereology, topology and systems theory [58] that are reusable modules according to which an ontology is organized. The final layer is comprised of application specific ontologies such as structure of object (i.e., AEC/FM specific material, geometry) and properties. The application layer refines the ontology to be used for precast model exchanges, by adding classes and relations for practical application of the ontology. In this case, the precast application ontology is built from (i) components, (ii) connections, (iii) system, (iv) placement, (v) material, (vi) geometry and (vii) requirements ontology. The components ontology provides the definitions for representing components in a building model and their part-whole decomposition.

Topology provides the is-connected-to relationship for connections between the components. On top of the component and connection, system ontology defines the aggregation of individuals into a system. A system provides functionality and behavior that are functions of its components and may have definitions of well-formed-ness. In order to complete the structure of a system, we have representation (geometry), material association, and placement. The functional aspects are defined in the requirements ontology.

The application ontology is built on top of engineering ontologies. The building element ontology defines how building elements should be specified in general, in the form of a set of theories. A building element can be modeled using the above-defined engineering ontologies, which are a part of the application ontology. Depending on the needs we can define a building element ontology using components, connections, system, aggregations, and adding classes for requirements, placement, and geometry. The following paragraphs provide excerpts of the ontology definitions. A complete reference on the ontology definitions can be found in [22].

\section{Ontology Definitions}

The component ontology defined in this research follows the theory of mereology, which is defined as the science or theory of parts, and is used to describe the part-of relation and its properties [58, 60]. The components ontology is used to represent the components in a building model and their part-whole decomposition. A component is a general concept that encompasses all individual elements used to describe the structure of an object. A component is part of an assembly and is considered to be atomic if it cannot be decomposed into any further parts. Assemblies can be made up of atomic components or smaller assemblies. Part-whole relationships are of two types, namely, simple part-of, and proper part-of relations. Part of is the general relationship that covers all the individuals in this ontology, whereas proper part-of restricts this relationship using the weak supplementation principle [58]. This principle states that, when an individual has a proper part, it must have another proper part disjoint from the first. A perfect example is the slab-beam aggregation (see Figure 5). A slab is the aggregation of individual beams, which means that beams are proper parts of the slab. In comparison, the project-site- building-building storyspace hierarchy is a simple part-of relationship. Moreover, in the case of proper part-of relationship, the geometry of the parent is the resulting sum of the individuals. This is expressed by the following definition, where $x$ and $y$ are parts of another shape:

$\mathrm{x}<\mathrm{y} \equiv \mathrm{x} \ll \mathrm{y} \vee \mathrm{x}=\mathrm{y}($ ProperPart - of $)$

Transitivity also holds for part-of relationship. Transitivity states that when an individual is a proper partof a second individual that is a proper part-of a third individual, then the first is also a proper part-of the third (A part of B and B part of $\mathrm{C}$, then A part of C). For example, a building has slabs, slab has hollowcore), and hence building has hollowcore. This is expressed by the following definition: 
Transitivity can be used to define assemblies as being assembled from parts. Asymmetry makes it impossible to say that an individual is a proper part of itself. If $\mathrm{A}$ is a part of $\mathrm{B}$, then $\mathrm{B}$ is not a part of $\mathrm{A}$.

$\mathrm{x} \ll \mathrm{y} \supset \sim \mathrm{y} \ll \mathrm{x}$ (Asymmetry)

\subsection{Part-Whole relationships}

According to the weak supplementation principle, explained previously, when an individual has a proper part then it must have another proper part disjoint from the first, which means an individual cannot be distinguished from the sum of its parts. This is shown by the following equation:

$\mathrm{x} \ll \mathrm{y} \equiv \mathrm{x}<\mathrm{y} \wedge \sim \mathrm{y}<\mathrm{x}($ ProperPart - of $)$

An example satisfying this constraint is the building element being a proper part-of another building element such as slab aggregation. Slabs are a composition of individual precast pieces, such as hollow core, DT or solid slabs. The cut shapes of these components fit inside of the slab shape as shown in Figure 7, where a wash is cut into the slabs to manage water run-off. The shape of a slab is defined as a general purpose, because the top of the slab may not be planar owing to toppings and washes. Care should be taken to ensure that the slab shape and its components, when unioned together, have no spaces between (except for the hollow-core holes) but they may be overlapping. Figure 7 shows the two different mappings for building elements. Figure 8a shows the building element aggregated into another building element, which in turn could be aggregated into a bigger assembly. Figure $8 \mathrm{~b}$ shows the building element being contained in the spatial hierarchy. In Figure $8 \mathrm{~b}$ the RelatingObject refers to a slab entity with geometry, material, possible embeds that are within the slab itself, but not in its other components. The RelatedObject references each of the component beams in this slab. Slab component pieces are assumed to be mutually disjoint, without overlaps. Thus specific recommendations of shape are defined for each type of embedded beam.

Figure 7: Aggregation of individual components into a slab: showing beams and wash [22].

Figure 8: Example showing mapping of different relationships in IFC for building elements: (a) Building Element Aggregation and (b) Spatial Containment.

IfcBuildingElements may or may not be overlapping, while IfcSpatialContainment is always overlapping in a nested tree structure. These distinctions are critical for volumetric and other analyses but are currently not distinguished.

We can also have assemblies aggregated into bigger assemblies. It was shown that to qualify for the proper part-of relationship, the weak supplementation principle needs to be satisfied. Based on this principle we can say that for a proper part-of relation the geometry of the parent will be the combined geometry of its parts. Accordingly, the relationship in Figure 8a satisfies this whereas Figure 8b fails. Whereas, in the case of the relationship in Figure $8 \mathrm{~b}$, the geometry of the spatial structure container cannot be deducted from the aggregation of the building elements in the space. This is a very important consideration that needs to be taken into account for calculating spaces. Appropriately, this part-ofrelation is a different type where the proper-part-of does not apply.

\subsection{Spatial Rules}

Definition of a model view requires multiple mechanisms to properly define the needed building semantics, including subschema specification and also additional rules defined at the instance level. IFC 
incorporates these, referring to them as business rules. Previous research has summarized the needs for development of a rule checking system as below [61]:

1. A method for mapping the terms in formally written statements into the logic of a testing condition,

2. Enriched data objects capable of deriving properties and relations needed to respond to rule queries

The authors believe that such efforts should be based on the development of an ontology of objects, properties, and relations for the relevant building domain [61]. Based on ontological definitions implemented in this research it can be shown that the first condition can be satisfied. For example, in a building-project model to be exchanged, there are three different types of spaces depicted based on their functionality - a lobby, elevator shaft, and laydown yard. Even though the space names are different, in each of the three cases, for an importing application they are all the same as they use IfcSpace as the common entity to represent the functional space. By default IFC associates space at building story level as shown in Figure 9a, in the case of a lobby area. However, based on the representational semantics from ontology, a reasoning system is able to differentiate between the functional spaces as shown in Figure 9(b). The lobby and elevator shaft are classified as internal so they can be at the building story level, whereas laydown yard is associated at site level (Figure 9c). Moreover, due to the additional constraint that elevator shaft traverses multiple floors, it is associated at building level (Figure 9b). Such reasoning capabilities are used for applications such as automated building code checking and validation.

\subsection{Overlapping Volumes}

Overlap and disjointness are defined as sharing a common part or the negation of this condition, as expressed by the following definitions. An individual overlaps another means that both are a part of the other. The following expressions define these:

$\mathrm{x} \circ \mathrm{y} \equiv \exists \mathrm{z}, \mathrm{z}<\mathrm{x} \wedge \mathrm{z}<\mathrm{y}($ Overlap)

$\mathrm{x} \mid \mathrm{y} \equiv \sim \mathrm{x} \circ \mathrm{y}($ Disjointness)

Figure 10a shows a precast beam-column-spandrel system, which is common in the precast domain. In this case there cannot be any overlap between these entities, which necessitates that the common part needs to be associated with one of the relating elements, as shown in Figure 10b and Figure 10c.

Figure 9: Example showing mapping of different spatial containment representation hierarchies based on the semantics read from an OWL/XML representation for spatial validation: (a) lobby associated at a building-story level, (b) elevator shaft associated at a building level and (c) laydown yard space directly associated at the site level.

Figure 10: Disjointness and overlap in a precast beam-column-spandrel system: (a) realization of a precast beam-column-spandrel system in a CAD tool, (b) beam connected to a column with common surface on beam, and (c) beam connected to a column with common surface on column.

Overlapping classifies proper part-of relationships into two classes here. Those that allow overlapping and those that do not. For example, a doubletee beam can be a proper part of slab, but does not allow overlap. Whereas, reinforcing is a proper part of beam but allows overlapping. Overlap can be checked by taking binary product of two individuals as expressed in the following definition.

$\mathrm{x} \times \mathrm{y}($ Binary Product) 
For non-overlapping individuals the above equation is invalid. For example, a beam is resting on a column and in precast these two individuals are not supposed to overlap. Hence, they cannot have a dot product, and therefore the shared part has to be assigned only to one of the individuals. The binary sum is the individual that encompasses at least one of $\mathrm{x}$ and $\mathrm{y}$. The difference is the individual, which is a proper part of $\mathrm{x}$ but does not share a part with $\mathrm{y}$. The following expressions define this:

$\mathrm{x}+\mathrm{y}($ Sum $)$

$\mathrm{x}-\mathrm{y}$ (Difference)

\subsection{Feature based Modeling}

Feature additions and subtractions in IFC are examples where sum and difference are checked. Sum is implemented by a Boolean addition to a precast piece, such as a corbel. Difference is implemented by Boolean subtraction and can be used for voids. Discrete accessory being a proper part-of a building element is an example of a simple part-of relationship that allows overlaps. A similar example is voids (opening) introduced in a building element in order to connect the two DTs using a seam connection. The IfcOpeningElement represents this void, which is a feature shape modifier. The connection component fills this void and is related through the IfcOpeningElement.

\subsection{Connections and Systems}

Any individual from the component ontology can be elevated to the level of type. Type is an object defined generically, such as a manufactured product, including small production runs of the same design, even when carried out on site. Instances of the type are related using the type-of relationship. Connection ontology provides the connections between objects by means of the is-connected-to relationship. The relationship in system aggregates individuals into a system. Figure 11a shows a precast system with a beam $(\mathrm{X})$ resting on a column $(\mathrm{Y})$. The beam is supported using a corbel $(\mathrm{Z})$ and the connection is completed using a bearing plate (R). According to IFC definitions, corbel is a feature added to the column and bearing plates are discrete accessories and distinguished using Standard Type Designation. IFC differentiates between their functional behaviors of ontology definitions as shown in Figure 11b. By enforcing the correct relations for the feature addition and the connection realization, the corbel and the bearing plate are clearly differentiated. The bearing plate and corbel are defined as the realizing element In addition the corbel is a feature addition to the column.

Figure 11: Connection between elements using a realizing element: (a) realization of a beam-column connection using a corbel and base plate in a CAD tool and (b) illustration of Ontology definition.

\section{7. applications of ontology definitions}

\subsection{Some comparison of component ontology and the IFC schema}

IFC is based on EXPRESS [62], which is a representational language, and STEP library definitions [63]. The STEP standard is known to be highly expressive but lacks a formal definition of its concepts [64]. Hence, semantic problems arise when explicit background information about entities is not available. Similar to many data schemas, IFC is highly redundant, offering multiple ways to define objects, relations and attributes. These issues along with the evolutionary nature of IFC prompted the authors to perform an analysis of the hierarchical structure of IFC with special focus on building elements and components.

Figure 12: IFC schema structure for building elements.

The objective of this exercise was to identify anomalies with respect to the classification structure of IFC and to propose a clean-up of the same. Figure 12 shows graphically the inheritance hierarchy of the IFC schema for elements. Global elements are subtypes of IfcBuildingElement but not shown (for economy of figure size) They may be IfcColumn, IfcBeam, IfcWall, IfcCurtainwall, IfcMember, IfcRamp, IfcStair, IfcRoof, IfcFooting, IfcPile, IfcSlab, and IfBuildingElementProxy and are classified as subtypes of 
IfcBuildingElement. Ontologically, they are all elements located within an element of the Spatial Hierarchy. Other elements are classified similarly but are located locally, relative to other BuildingElements. These are color-coded light green. Other subtypes of BuildingElement modify the geometry on BuildingElements subtypes: IfcFeatureElement and IfcCovering. Other components and parts are shown in Figure 12 with varying coherence regarding their implicit classification rules (suggested in the figure by their color). One type of shape modification is a connection relating elements together. All components are subtypes of IfcElement, and thus have the same base properties and inverse relations. For example, reinforcing tendons, mechanical fasteners, and discrete accessories such as nuts, bolts are all components that can be counted and can be represented as arrays. However, they are classified under different parent classes as seen in Figure 12.

The anomalous examples are probably the result of the evolutionary definitions for elements defined in different releases of IFC. Such anomalies make the definition of a model view arbitrary in nature. As can be seen, building components in IFC appear to have an ad hoc structure. In programming languages, abstract types are used for classification, to represent common behavior or implementation. The classification logic of the abstract types in IFC is not clear. As can be seen in Figure 12, IFC components include classifications of IfcFeatureElement, IfcBuildingElement (those elements that are not globally placed, but rather are parts of other elements), and IfcElementComponent and the subtypes of IfcBuildingElementComponent and IfcReinforcingElement.

Of these IfcBuildingElement also have add-on components to define stairs, railings and ramps. These we classified together as being: inter-floor or modifying inter-floor elements (stairs and Ramps), and are associated with other building elements. IfcFeatureElementSubtraction and IfcFeatureElementAddition modify building elements using the Boolean operations. In IFC, they may be merged as B-Rep or articulated for measurement or editing. IfcWindows and IfcDoors provide fillers to IfcFeatureElementSubtraction that create openings in walls or ceilings. IfcFastener and its subtype, IfcMechanicalFastener, are subtypes of IfcElementComponent. IfcFastener is most often a realizing element for IfcRelConnectsWithRealizingElements that provide elements that are the physical realization of a connection. This may include plates, bolt assemblies, welds and other attachment types (some not yet defined in IFC). In general, these elements are defined ad hocly; IfcPlate is a building element, and also an enumerated type of IfcDiscreteAssembly. They should be consistently defined in the same family of IfcFastener. To provide a more precise definition of IfcDiscreteAssembly, we propose they be only premanufactured elements identifiable with a product identifier, with fixed shape represented as a B-Rep.

IfcCovering deals with the finishes and covering on building elements or their components and should be associated with the components they modify (similar to IfcFeatureAddition and Subtraction). IfcReinforcingElement is a common subtype of IfcBuildingElementComponent with IfcBuildingElementPart. While the function of IfcReinforcingElement and its subclasses are clear, their relation to IfcBuildingElementPart is not. The parts are defined in the published examples of parts of a wall, such as a wythe in a masonry wall, or an insulation layer, or wood framing in a wall. These also have other classifications, such as IfcMaterialLayerSet. We propose that IfcBuildingElementPart be consistently used for features that are significant parts of building elements, such as the layers of IfcWall or IfcSlab. They are typically composed using IfcRelAggregates into an assembly for the wall or slab. IfcBuildingElementProxy is an ad hoc category that can be used to define any component not otherwise classified.

This analysis articulates the point that the classification structure of IFC is defined in an ad hoc manner. A logic-based approach is needed to support extension and reliability of the work of future generations of IFC model extenders., Future growth by diverse individuals should be anticipated, so the basis of the IFC structure can consistently evolve. The following section explores the application of ontology definitions and constraints to arrive at a better and semantically consistent classification structure. 


\subsection{Defining a new classification structure based on ontology definitions}

Buildings are made up of objects. But what is an object? According to the ontology definition, an object is any physical or information object in a model exchange. However, object attributes contain general information about the individual, such as aggregation, connected supports, a process and many others. The IFC object definition is extended to include processes, controls, and actors as well, which will have to be more logically resolved with IfcObject in the future. The structural ontology uses IfcProduct to qualify three relationships namely (a) has representation, (b) has material association, and (c) has placement. An object has material associated with it, however the material requirement should be extended and defined in the requirements ontology. Geometry is an area that has been well developed over the years [65] and for purposes of this research we assume geometry is defined in a separate high-level ontology. Every product individual has a placement relationship and can be realized by three different mechanisms, namely, absolute placement, placement relative to a grid, and placement relative to another individual. The requirements ontology contains main concepts needed for the representation of the function and behavior of individuals. For example, the requirements for a precast piece can be decomposed into requirements related to performance, design criteria, production, delivery and erection methods.

These definitions are similar to the IFC definitions for IfcProduct. The focus of this comparative study begins at the IfcElement level and classifies the hierarchy based on definitions from the ontology. The scope of this study is limited to only those elements, which are related to the structure of a building and does not yet include entities such as mechanical, electrical, or plumbing equipment. A breadth first approach has been followed to divide the elements into two broad groups namely, (a) primary building elements, and (b) element components. Any building element that has its own existence in the spatial structure of the project is considered to be a primary building element. Whereas, those elements that are present to fulfill a functional role in relation to a primary building element fall under the category of element components. The element components can be load bearing or non-load bearing. Typing and creation of assembly are at the object level and should be allowed for both primary building elements and element components.

Based on the above definitions, the IFC schema is analyzed and only the following set of elements satisfy the requirements for primary building elements. This set is as follows:

PrimayBuildingElements = [IfcBeam, IfcChimney, IfcColumn, IfcCurtainWall, IfcDoor, IfcFooting, IfcMember, IfcPile, IfcRamp, IfcRoof, IfcSlab, IfcStair, IfcWall, IfcWindow, IfcProxyBuildingElement]

If the element components are to be classified further, then it can be based on the functional relationship they have with the associated primary building element. Accordingly, the element components are grouped as element parts, element accessories, and element modifiers. In many instances primary building elements can be a container object for aggregating different components. Element parts are such entities that can be aggregated into a primary building element. Examples of element parts are sandwich wall panels, stair and ramp flights, and coverings for slabs.

\section{ElementParts $=$ [IfcCovering, IfcRampFlight, IfcStairFlight, IfcBuildingElementPart, IfcDoor,} IfcWindow]

Accessories are those entities attached or embedded in a primary building element, but they cannot be aggregated in the same way as building element parts to the primary building element. Examples of element accessories are lifting hooks, fasteners, joints, and reinforcing. It should be noted that IFC does not have a specialized relationship currently to differentiate building element parts and accessories. Both are handled by the general IfcRelDecomposes relationship, which has the subtypes IfcRelAggregates and IfcRelNests. IfcRelAggregates, which is used for all aggregation relationships, is insufficient to 
distinguish between the building-element parts that are aggregated in a container from element components that are attached to an element. This is especially important in cases of volume calculations. For example, the volume of a slab is calculated considering the covering and other parts; whereas the volume of rebar is not subtracted. IFC documentation explains that such a dependency is implied and left to the computing application to decide. However, based on ontology definitions the strict constraints differentiate between the parts and accessories and place them in different classification groups.

The third type of element components is the element modifier. These are virtual elements used for feature-based modeling. These are treated as semantic objects but the only functional existence for such elements is for the purpose of modifying the shape of the primary building element. They make use of addition and subtraction operations to modify the geometry. Clearly, the use of aggregation relationship does not satisfy this scenario, as there is a difference between aggregating components that are equal parts, and features, which are subordinate parts. Feature additions and subtractions are already discussed in the ontology definitions section.

It can be further mandated that any entity in the primary building element family should have its globally unique identifier, whereas, the element components should not be assigned a globally unique id directly. Such a constraint also serves practical purposes since IFC is criticized for performance issues and assigning GUIDs, owner history and geometry to each and every element component is one of the main issues. We propose the introduction of an array object for element component, which can have a GUID and each element in the array, can be individual rebar or mechanical fasteners. Other attributes such as count of rebars can be derived by the application if such an array object is used. There are three main considerations for introducing an array object, namely (a) the model footprint, (b) the effort in identifying the array elements, and (c) the ability to modify the array object with new elements or varying spacing.

The ontology structure allows defining a more logical and consistent semantic structure to the building elements, defined as concepts. An important benefit of this classification is that, ideally based on their function, different users, as well as the software implementers can unambiguously define classes. Element classes should be disjoint, leading to a single implementation approach. Based on the above discussions and the ontology structure, a new element hierarchy is proposed for the IFC schema, which is shown in Figure 13. In the proposed hierarchy, individual instances of element components need GUIDs only if they have independent existence, not dependent upon another element. Otherwise, the element component has the ability to carry an array of elements, with the ObjectType attribute specifying the type of the element carried in the array. Each element in the array will have a placement and representation associated with it. To fully address the performance issues, the array object should be restricted to carry entities referring to same geometry. Additional properties are provided by attaching corresponding property sets. Such a re-structuring of the IFC schema will clean up the hierarchical structure in a semantic way and facilitate development.

Figure 13: Proposed schema structure for elements in the IFC schema based on the ontological definitions for Part-Of, Proper Part-Of and others defined in the text.

\section{Conclusion and Outlook}

This research outlines a possible foundation for the development and implementation of a new integrated framework that is expected to define a clearer and less ad hoc structure for IFC. The new structure, like the current IFC is extensible. In addition, the method and classifications of extensions are well defined. This research has shown the usefulness of ontologies for specifying consistent implementation structures using IFC. Formally specified ontologies enable the use of reasoning engines for verification and automatic classification. These functionalities can be utilized in the future to define new model views and also to check their well-formedness. 
The major findings, some limitations and future potential are explained with respect to the research questions presented in this paper.

How can we improve the consistency of IFC-based model exchanges across teams and domains? In order to support IFC implementations, the consistency of model views designed is an important criterion, lack of which is causing overhead for software developers and is inhibiting new IFC model view implementations. Product model schemas such as IFC are rich, but redundant. Based on the insights gathered from developing the Precast National BIM Standard and further analysis as part of this research, a new methodology based on ontological definition of the IFC schema is introduced. The definition of a Precast System Ontology is explained. Based on the analysis, it is shown that MVD development process needs to be transitioned from the current ad hoc manner to a more rigorous framework and/or methodology similar to the one explained in this research.

This research provides an analysis of IFC product model schema with specific focus on building elements and components. We identified discrepancies in the classification structure of the element components and their intended functionality. A new hierarchical structure for the building elements and components is suggested, based on the ontological definitions. In order to avoid ambiguities in current and future model exchanges it is imperative that a thorough analysis and clean-up of the schema hierarchy is necessary as shown in this research. This definition provides a classification structure for object-oriented modules to be developed on top of ontologies. Such modules are potentially more than an implementation module; they define a semantic unit to be defined in a model for exchange (identifying user intent and software capabilities).

Future work might include an ontology-based object-oriented library for model view development, testing, and certification. An ontology specifies how the application functionality is to be implemented and it serves roles similar to entity-relationship (ER) diagrams, object models, and object patterns. In the case of model exchanges, the ontology is intended to provide the structure of the model view. For this purpose, the ontology layer developed in this research is converted to an object-oriented class library. This additional layer of mapping is used for implementation. IFC entities, relations, attributes, etc. are mapped onto the application ontology developed in the previous phase. This allows ontology language, definitions and modeling representations such as IFC to stay invisible to the end user (similar to data hiding in object-oriented design). This significantly lowers the barriers for practitioners (software developers). This library is developed to be extensible and it is envisioned that future model views will be developed based on this library.

\section{Acknowledgments}

The work presented here was funded by the National Institute of Standards and Technology, grant number 60NANB9D9152, the Digital Building Laboratory at the Georgia Institute of Technology, and the Charles Pankow Foundation. All information presented is that of the authors alone.

\section{References}

[1] C. Eastman, P. Teicholz, R. Sacks, K. Liston, BIM Handbook: A guide to building information modeling for owners, managers, designers, engineers, and contractors, John Wiley \& Sons Inc., 2008.

[2] M. Gallagher, A. O'Connor, J. Dettbar, L. Gilday, Cost Analysis of Inadequate Interoperability in the US Capital Facilities Industry (NIST GCR 04-867), (2004).

[3] N. Young, S. Jones, H. Bernstein, J. Gudgel, The Business Value of BIM: Getting Building Information Modeling to the Bottom Line, Technical Report, The McGraw-Hill Companies, New York, (2009). 
[4] IAI-Tech, Industry Foundation Class (IFC) data model, Technical Report, BuildingSMART, 2003. http://buildingsmarttech.org/specifications/ifc-releases/summary (last accessed 10/23/2011).

[5] ISO, ISO/PAS 16739: 2005, Industry Foundation Classes. http://www.iso.org/iso/ (last accessed 10/23/2011).

[6] BuildingSMART, IFC Overview Summary, http://buildingsmarttech.org/specifications/ifc-overview, 2010 (last accessed 10/23/2011).

[7] A. Kiviniemi, Ten Years of IFC Development - Why are we not yet there?, in: CIB-W78 Keynote, Montreal, (2006).

[8] M. Venugopal, C.M. Eastman, R. Sacks, J. Teizer, Semantics of model views for information exchanges using the industry foundation class schema, Advanced Engineering Informatics, 26(2) (2012) 411-428.

[9] C.M. Eastman, I. Panushev, R. Sacks, M. Venugopal, V. Aram, R. See, A Guide for Development and Preparation of a National BIM Exchange Standard, Technical Report, PCI, Charles Pankow Foundation, Georgia Instutute of Technology and Technion, (2011).

[10] T. Gruber, et al., A translation approach to portable ontology specifications, Knowledge acquisition, Special issue in knowledge monitoring, 5(2) (1993) 199-220.

[11] NBIMS, National Building Information Modeling Standard, Version 1.0 Part 1 Overview, Principles, and Methodologies, Technical Report, National Institute of Building Sciences (NIBS), http://www.buildingsmartalliance.org, 2007 (last accessed 6/10/2010).

[12] V. Aram, C. Eastman, R. Sacks, I. Panushev, M. Venugopal, Introducing a new methodology to develop the information delivery manual for AEC projects, in: Proceedings of the CIB-W078 2010: 27th International Conference, Cairo, (2010).

[13] C.M. Eastman, Y.S. Jeong, R. Sacks, I. Kaner, Exchange model and exchange object concepts for implementation of national bim standards, Journal of Computing in Civil Engineering, 24(1) (2010) 2534.

[14] J. Hietanen, IFC model view definition format, International Alliance for Interoperability, 2006.

[15] C.M. Eastman, R. Sacks, I. Panushev, M. Venugopal, V. Aram, Precast concrete BIM standard documents: model view definitions for precast concrete, PCI-Charles Pankow Foundation, http://dcom.arch.gatech.edu/pcibim/documents/Precast_MVDs_v2.1_Volume_I.pdf (last accessed on 6/20/2010) (2010).

[16] ACI, Cast-in place National BIM Standard, Technical Report, American Concrete Institute, http://dcom.arch.gatech.edu/acibim/ (last accessed on April 22, 2012) (2012).

[17] AISC, AISC BIM Standard Project, Technical Report, American Institute of Steel Construction, http://dcom.arch.gatech.edu/aisc/ (last accessed on April 22, 2012) (2012). 
[18] J. Hietanen, Information delivery manual: Guide to components and development methods, http://iug.buildingsmart.org/idms/development/IDMC_004_1_2.pdf (last accessed on April 22, 2012) (2006).

[19] GTDS, IFC Certification 2.0: Global Testing documentation server, Technical Report, buildingSMART, http://gtds.iabi.eu (last accessed on May 14, 2012) (2012).

[20] R. See, IFC Solutions Factory: Model View Definitions Site, Technical Report, BuildingSMART, 2010. http://www.blis-project.org/IAI-MVD/ (last accessed on 6/20/2011) (2011).

[21] J. Beetz, J. Van Leeuwen, B. De Vries, IfcOWL: A case of transforming EXPRESS schemas into ontologies, Artificial Intelligence for Engineering Design, Analysis and Manufacturing, 23 (2009) 89101.

[22] M. Venugopal, Formal Specification of Industry Foundation Class Concepts using Engineering Ontologies, Ph.D. thesis, Georgia Institute of Technology, Atlanta, GA., http://smartech.gatech.edu/handle/1853/3739 (last accessed on April 22, 2012) (2011).

[23] J. Garrett, S. Fenves, D. Stasiak, A www-based regulation broker, CIB REPORT (1996) 219-230.

[24] V. Liang, J. Garrett, Java-based regulation broker, Journal of Computing in Civil Engineering, 14(2) (2000) 100-108.

[25] D. Stasiak, J. Garrett Jr, S. Fenves, A broker for tracking, delivering and using regulations over the world wide web, in: Electronics and the Environment, 1996. ISEE-1996., Proceedings of the 1996 IEEE International Symposium on, IEEE, (1996) 293-297.

[26] C. Caldas, L. Soibelman, Integration of construction documents in IFC project models, in: K.R. Molenaar, P.S. Chinowsky (Eds.), Proceedings of Construction Research Congress, (2003) 1-8.

[27] C. Caldas, L. Soibelman, L. Gasser, Methodology for the integration of project documents in modelbased information systems, Journal of Computing in Civil Engineering 19(1) (2005) 25-33.

[28] F. Baader, D. Calvanese, D. McGuinness, P. Patel-Schneider, D. Nardi, The description logic handbook: theory, implementation, and applications, Cambridge Univ Pr, (2003).

[29] D. Fensel, I. Horrocks, F. Van Harmelen, S. Decker, M. Erdmann, M. Klein, OIL in a nutshell, Knowledge Engineering and Knowledge Management Methods, Models, and Tools, Lecture Notes in Computer Science, Springer Verlag, 1937 (2000) 1-16.

[30] A. Gomez-Perez, M. Fernandez-Lopez, O. Corcho, Ontological engineering: with examples from the areas of knowledge management, e-Commerce and the Semantic Web, Springer Verlag, 2004.

[31] T. Gruber, G. Olsen, An ontology for engineering mathematics, in: Fourth International Conference on Principles of Knowledge Representation and Reasoning, Gustav Stresemann Institut, Bonn, Germany, Morgan Kaufmann, (1994) 241-245.

[32] G. Van Heijst, A. Schreiber, B. Wielinga, Using explicit ontologies in kbs development, International Journal of Human Computer Studies, 46 (1997) 183-292. 
[33] R.J. Brachman, J.G. Schmolze, An overview of the kl-one knowledge representation system, Cognitive Science, 9 (1985) 171-216.

[34] M. Genesereth, R. Fikes, Knowledge interchange format, version 3.0 reference manual, Stanford University, Computer Science Department, 1992.

[35] A. Farquhar, R. Fikes, J. Rice, The Ontolingua Server: a tool for collaborative ontology construction, International Journal of Human-Computer Studies, 46 (1997) 707-727.

[36] M. Kifer, G. Lausen, F-logic: a higher-order language for reasoning about objects, inheritance, and scheme, SIGMOD Rec. 18 (1989) 134-146.

[37] J. Angele, G. Lausen, Ontologies in f-logic, Handbook on Ontologies, Springer Verlag (2004) 29-50.

[38] Y. Umeda, H. Takeda, T. Tomiyama, H. Yoshikawa, Function, behaviour, and structure, Applications of Artificial Intelligence in Engineering, 1 (1990) 177-193.

[39] B. Chandrasekaran, Functional representation: A brief historical perspective, Applied Artificial Intelligence, 8 (1994) 173-197.

[40] B. Chandrasekaran, R. Milne, Reasoning about structure, behavior and function, SIGART Bull, (1985) 4-55.

[41] V. Sembugamoorthy, B. Chandrasekaran, Functional representation of devices and compilation of diagnostic problem-solving systems, in Experience, memory and Reasoning, J. Kolodner and C. Riesbeck (eds.), Lawrence Erlbaum Associates, (1986) 47-73.

[42] A. Goel, S. Rugaber, S. Vattam, Structure, behavior, and function of complex systems: The structure, behavior, and function modeling language, Artificial Intelligence for Engineering Design, Analysis and Manufacturing, 23 (2009) 23-35.

[43] M. Fischer, J. Kunz, The scope and role of information technology in construction, CIFE Technical Report \#156, (2004) 1-17.

[44] I. Smith, Intelligent computing in engineering and architecture, Springer-Verlag GmbH., 2006.

[45] Q. Yang, Y. Zhang, Semantic interoperability in building design: Methods and tools, ComputerAided Design, 38 (2006) 1099-1112.

[46] M. Böhms, P. Bonsma, P. Willems, A. Zarli, M. Bourdeau, E. Pascual, G. Storer, S. Kazi, M. Hannus, J. Sedano, et al., The SWOP Semantic Product Modelling Approach, Technical Report STRP NMP2-CT-2005-016972 "SWOP”, TNO, 2008.

[47] BuildingSMART, mvdXML Format, http://buildingsmarttech.org/specifications/mvd-overview, (last accessed on April 22, 2012), 2010.

[48] mvdXML, mvdXML Schema, buildingSMART International, (http://www.buildingsmart-tech.org/) (last accessed on April 22, 2012), (2012).

[49] G. Booch, J. Rumbaugh, I. Jacobson, Unified Modeling Language User Guide, The Addison-Wesley Object Technology Series, Addison-Wesley Professional, 2005. 
[50] J. Arplrez, O. Corcho, M. Fernandez-Lopez, A. Gomez-Perez, Webode in a nutshell, AI magazine, 24(3) (2003) 37-47.

[51] A. Maedche, B. Motik, L. Stojanovic, R. Studer, R. Volz, Ontologies for enterprise knowledge management, IEEE Intelligent Systems, 18 (2003) 26-33.

[52] Y. Sure, M. Erdmann, J. Angele, S. Staab, R. Studer, D. Wenke, OntoEdit: Collaborative Ontology Development for the SemanticWeb, The Semantic Web-ISWC 2002, Springer, (2002) 221-235.

[53] M.A. Musen, Automated support for building and extending expert models, Machine Learning, 92 (1990) 101-129.

[54] J.H. Gennari, M.A. Musen, R.W. Fergerson, W.E. Grosso, M. Crubezy, H. Eriksson, N.F. Noy, S.W. $\mathrm{Tu}$, The evolution of Protege: an environment for knowledge-based systems development, International Journal of Human-Computer Studies, 58 (2003) 89-123.

[55] E. Hjelseth, N. Nisbet, Exploring semantic based model checking, in: CIB-W078 Conference in Cairo, (2010).

[56] M. Venugopal, C.M. Eastman, R. Sacks, Configurable model exchanges for the precast/pre-stressed concrete industry using semantic exchange modules (sem), International Conference on Computing in Civil Engineering, (2012) 269-276.

[57] T. Tudorache, Employing Ontologies for an Improved Development Process in Collaborative Engineering, Ph.D. thesis, Berlin University of Technology, Berlin, Germany, 2006. (http://opus.kobv.de/tuberlin/volltexte/2006/1437/) (last accessed on April 22, 2012).

[58] W. Borst, Construction of engineering ontologies for knowledge sharing and reuse, Ph.D. thesis, Centre of Telematica and Information Technology, Universiteit Twente: Enschede, The Netherlands, 1997.

[59] C. Masolo, S. Borgo, A. Gangemi, N. Guarino, A. Oltramari, L. Schneider, The WonderWeb library of foundational ontologies, WonderWeb Deliverable D 17 (2002).

[60] P. Simons, Parts: A study in ontology, Clarendon Press, USA, 1987.

[61] C. Eastman, J. Lee, Y. Jeong, J. Lee, Automatic rule-based checking of building designs, Automation in Construction, 18 (2009) 1011-1033.

[62] D.A. Schenk, P.R. Wilson, Information Modeling the EXPRESS Way, Oxford University Press, USA, 1994.

[63] C.M. Eastman, Overview of ISO STEP, Technical Report, Digital Building Lab, Georgia Tech, 2006. (http://bim.arch.gatech.edu/contents/OverviewIFC3[1].htm) (May 14, 2012).

[64] N. Guarino, S. Borgo, C. Masolo, Logical modelling of product knowledge: towards a well-founded semantics for step, in: Proceedings of European Conference on Product Data Technology (PDT Days 97), Sophia Antipolis, France, Citeseer, 1997. 
[65] L.A. Piegl, Fundamental Developments of Computer-Aided Geometric Modeling, $1^{\text {st }}$ edition, Academic Press, 1993.

[66] Associated General Contractors, Level of Development Specification For Building Information Models, Version: 2014 Issued: Dec. 30, 2014. 


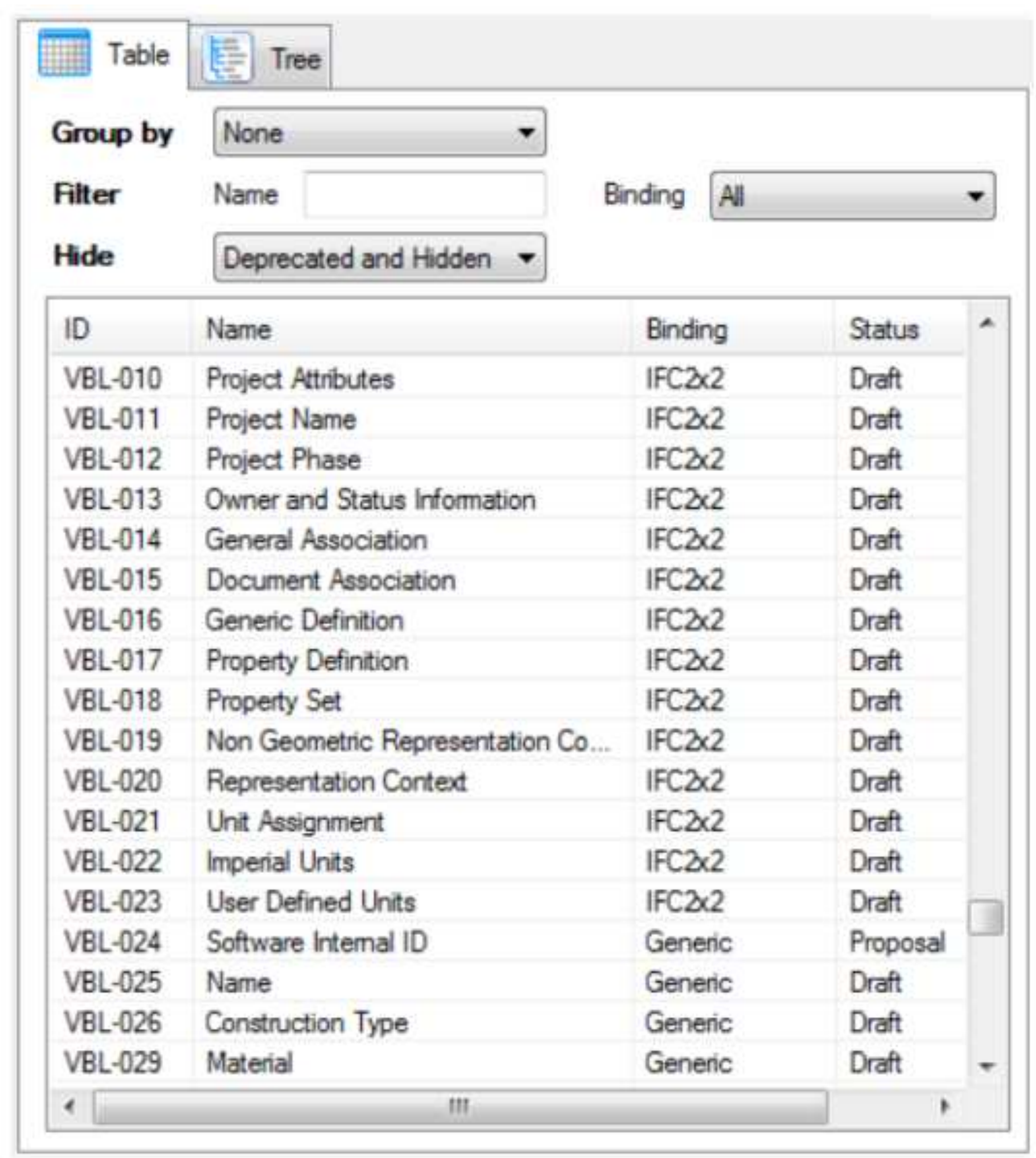




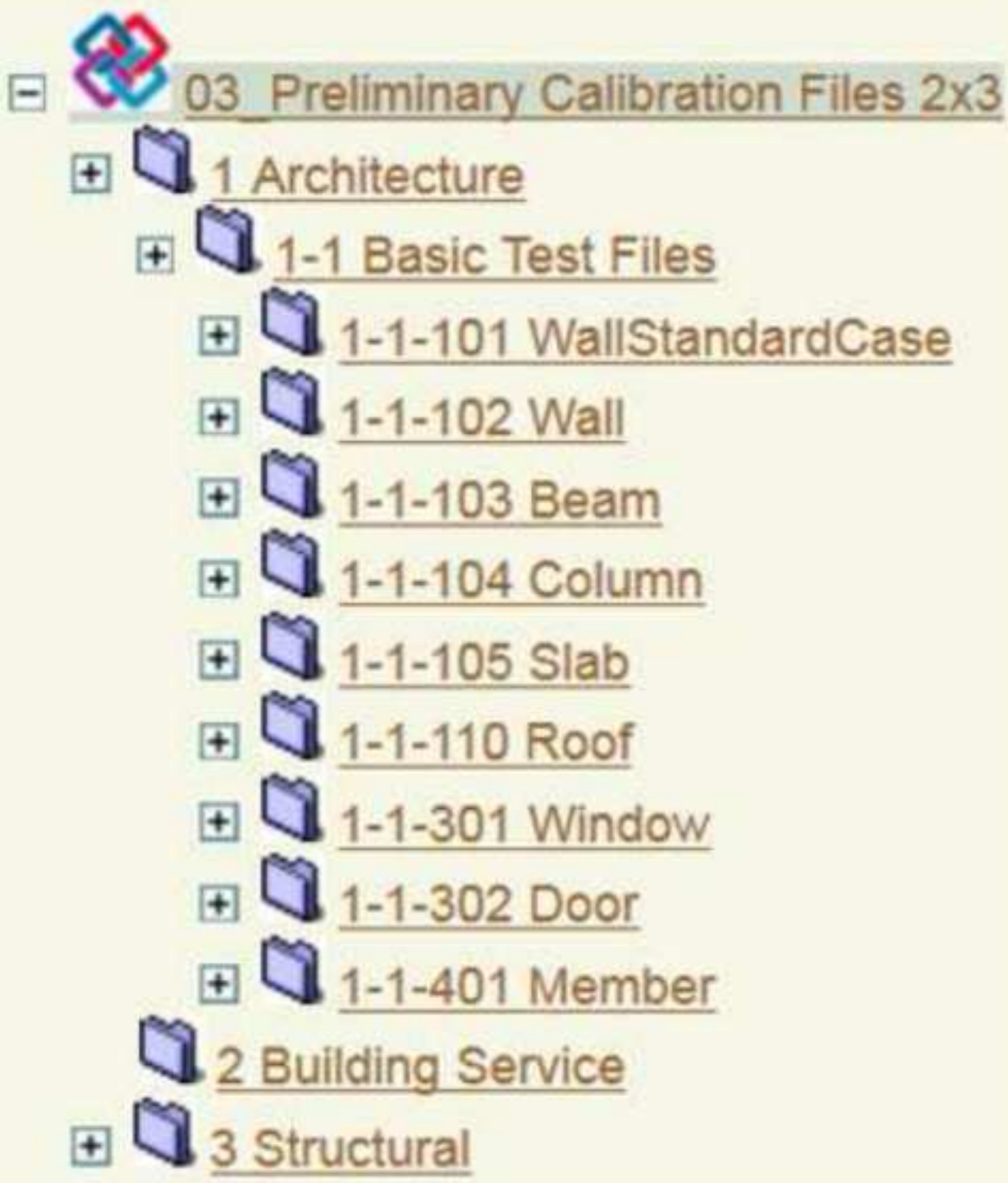

- 0303 Preliminary Calibration Files $2 \times 3$

\pm 1 Architecture

+ 1 1-1 Basic Test Files

t) 1-1-101 WallStandardCase

t) 1-1-102 Wall

+$]_{1-1-103}$ Beam

+) 1-1-104 Column

$\mp Q_{1-1-105 \text { Slab }}$

+] 1-1-110 Roof

† 1-1-301 Window

t) 1-1-302 Door

† 1-1-401 Member

2 Building Service

๑ 3 Structural 


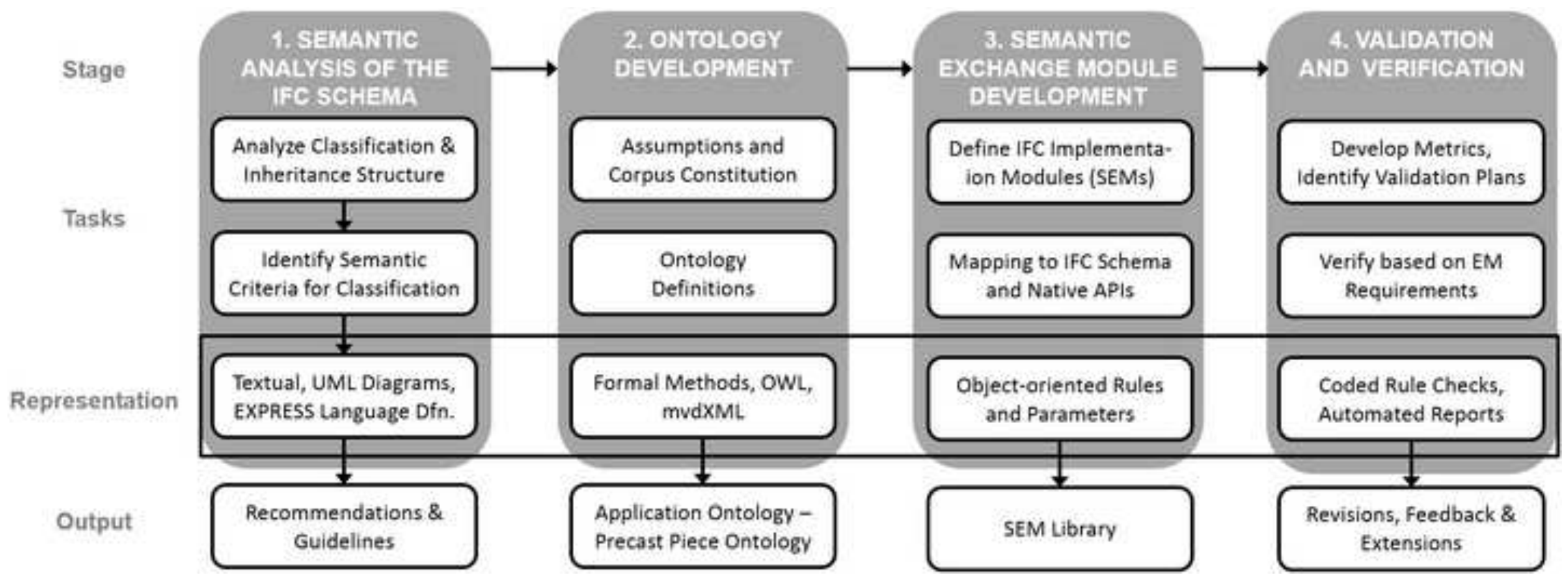


(a)

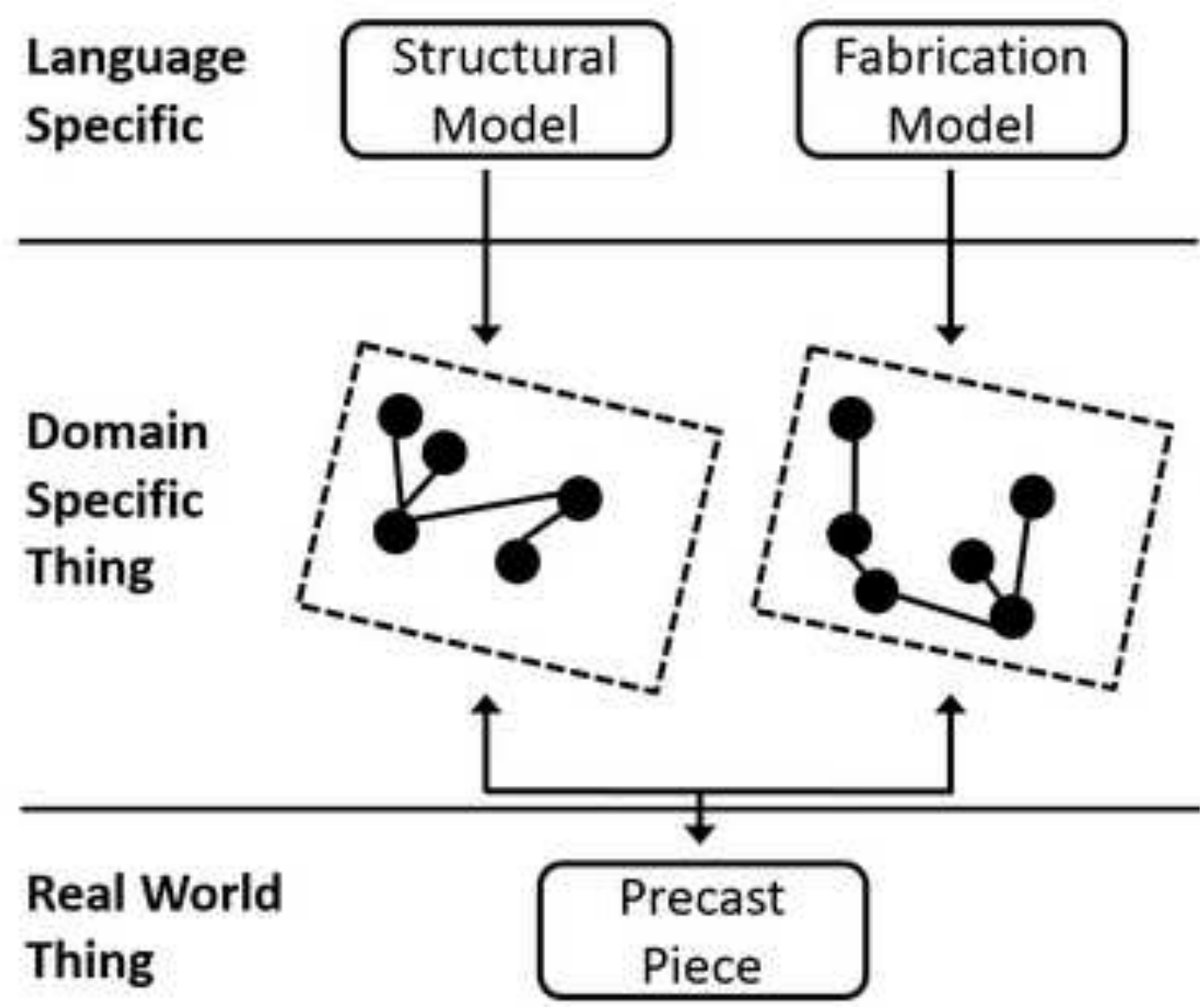

View 2 (b)

(c)
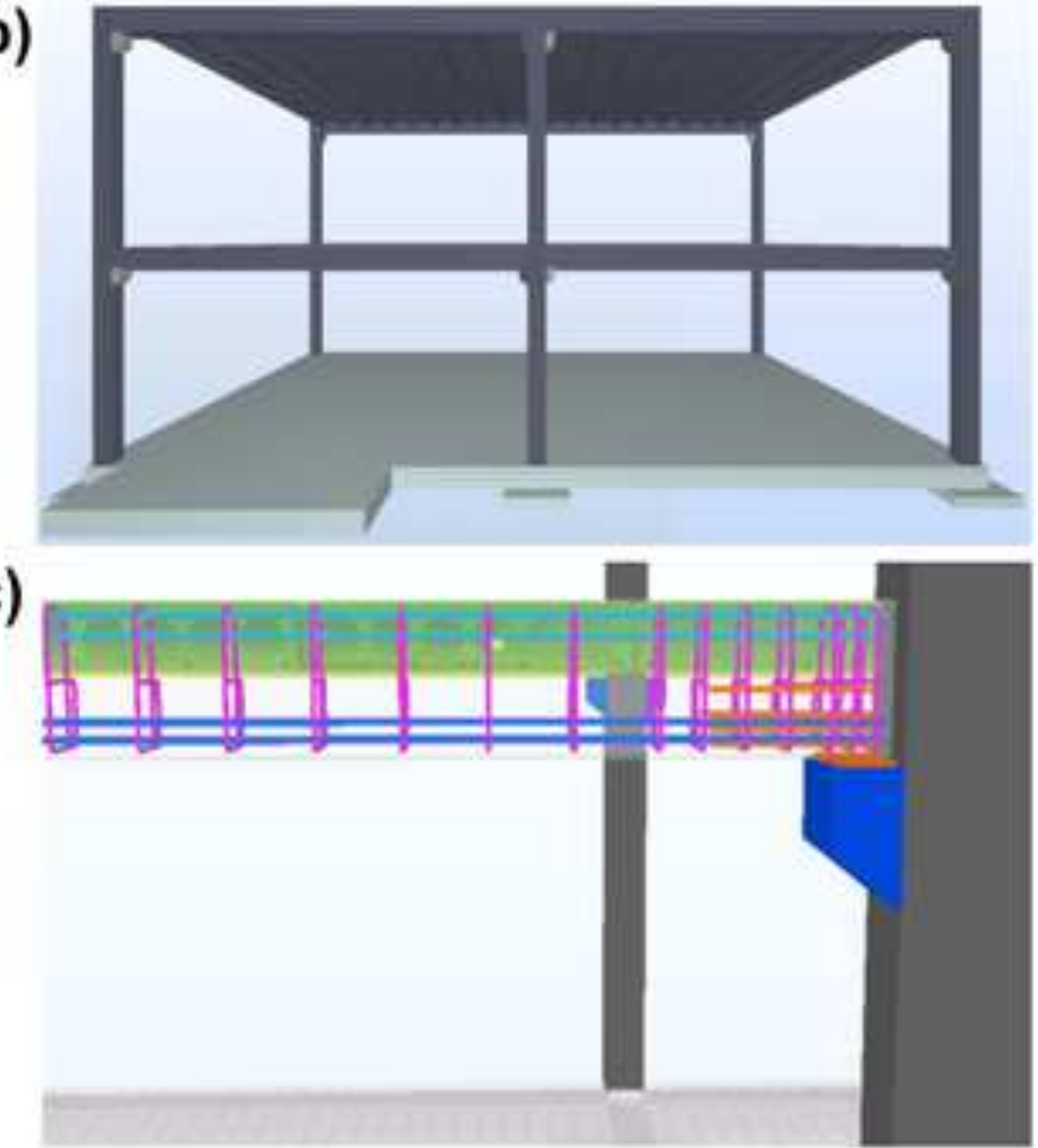


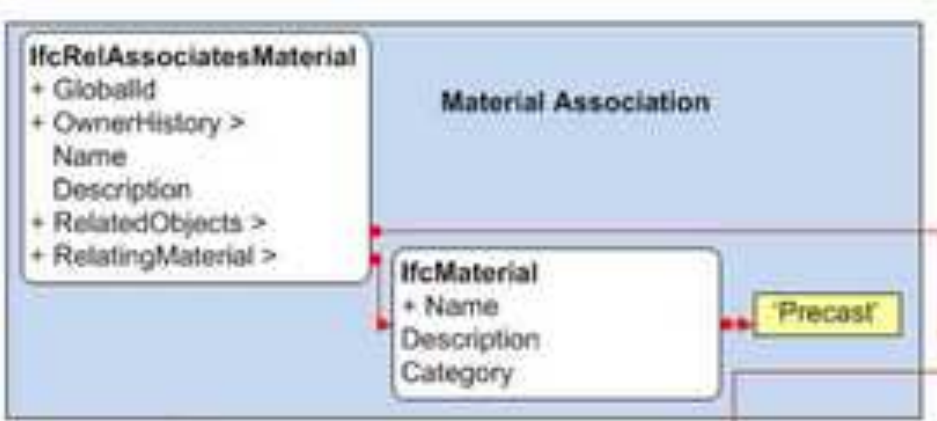

\section{UfcBuildingElement}

+ Gioballd

+ OmnerHistory $>$

Name

Description

Objectiype

ObjectPlacement>

Representabon?

Tag

- (iNV) HasAseciations

- (INV) isDocomposedey

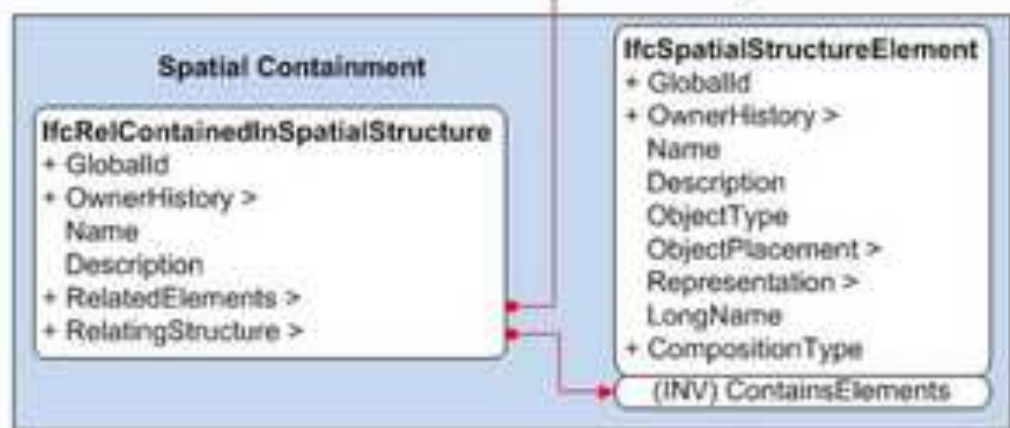

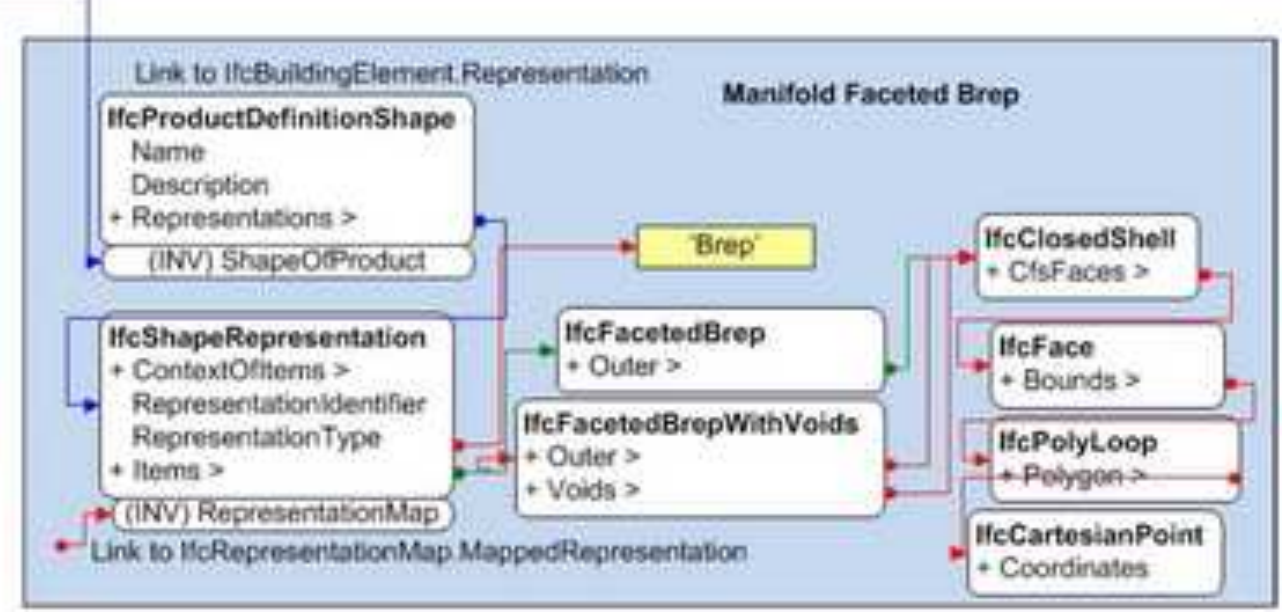

\begin{tabular}{|c|c|c|}
\hline \multirow[b]{3}{*}{$\begin{array}{l}\text { IfeLocalPlacement } \\
\text { PlacementRerto s } \\
\text { + RelativePlacement s } \\
\text { (INV) Placescobiects }\end{array}$} & liute Placem & \multirow{2}{*}{$\begin{array}{l}\text { HeCarteslanPoint } \\
\text { - Coordinates }\end{array}$} \\
\hline & & \\
\hline & $\begin{array}{l}\text { HeAxis2Placement30 } \\
\text { + Location > } \\
\text { Auis > } \\
\text { RerDirection > }\end{array}$ & $\begin{array}{l}\text { - Coordinates } \\
\text { HeDirection } \\
+ \text { DirectionRatios }\end{array}$ \\
\hline & & $\begin{array}{l}\text { HeDirection } \\
\text { + DirectionRatios }\end{array}$ \\
\hline
\end{tabular}



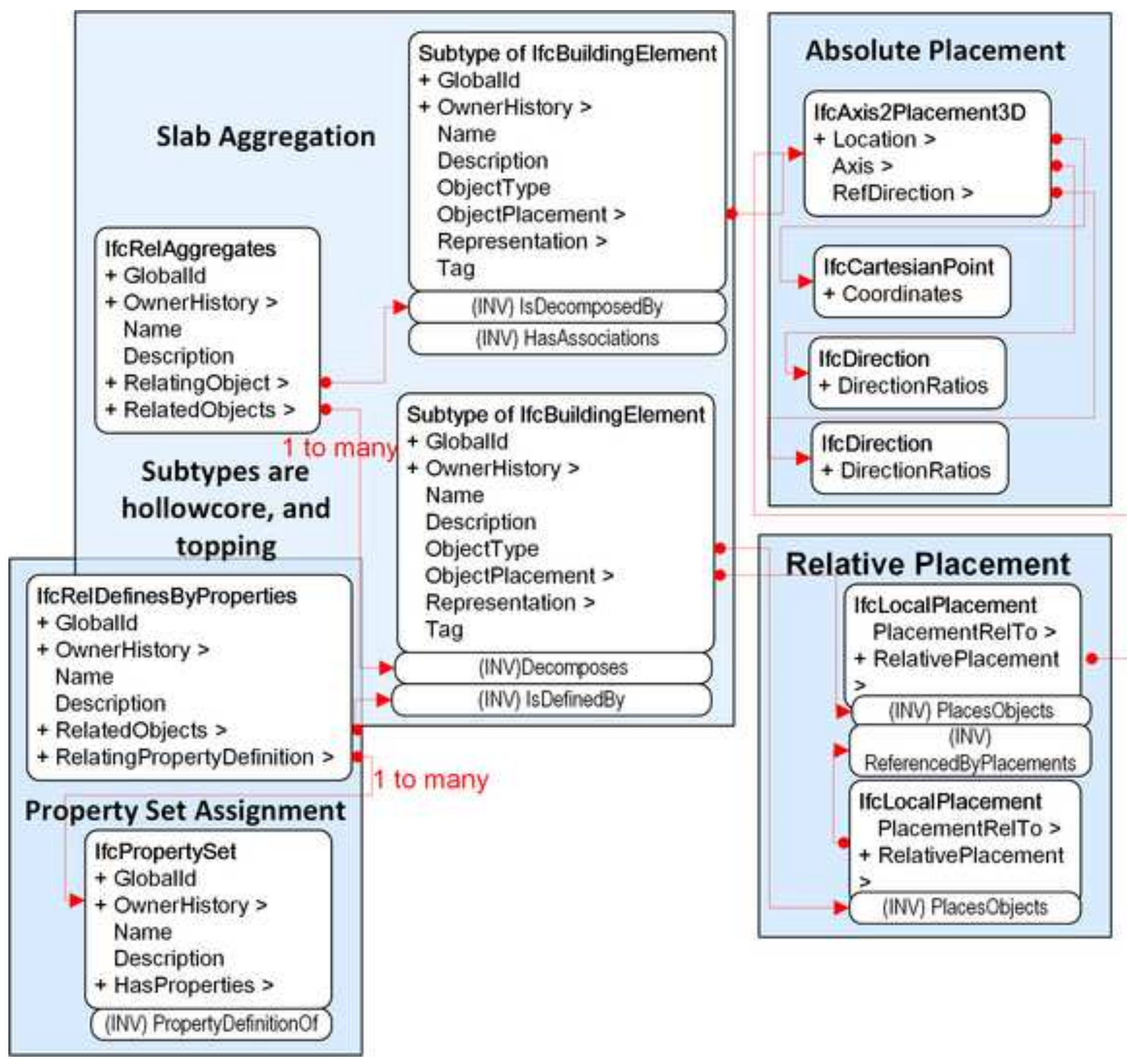

Name

Subtype of IfcBuilding Element

Globalld

Name

Description

ObjectType

ObjectPlacement >

Representation >

+ Globalld

OwnerHistory >

atedobjects

IfcPropertySet

+ Globalld

Name

+ HasProperties > 
Figure 06

Top-level Ontology (Abstract)

\section{Thing}

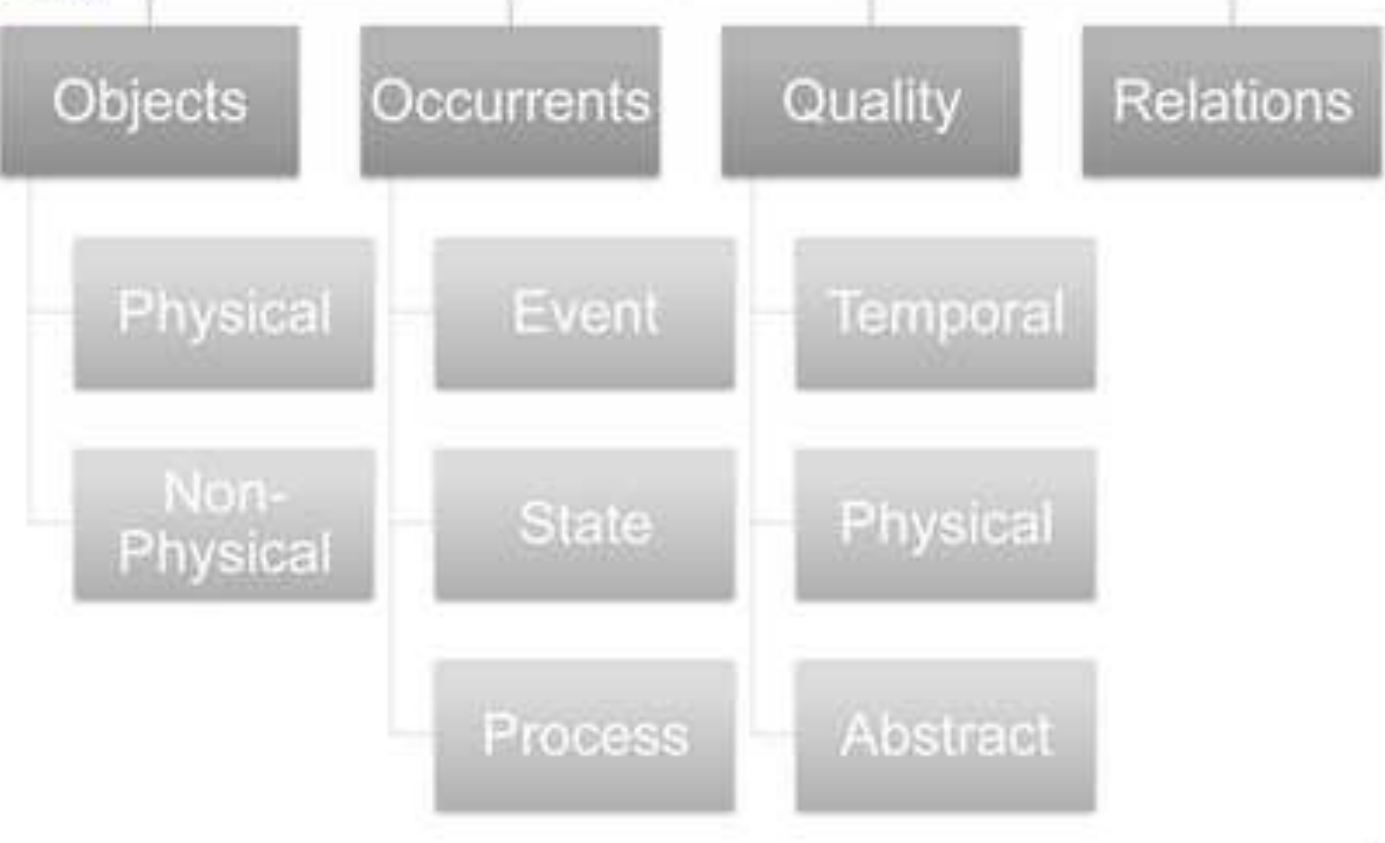

\section{Engineering Ontologies} (Domain Specific)

Application Ontology (Context Specific)
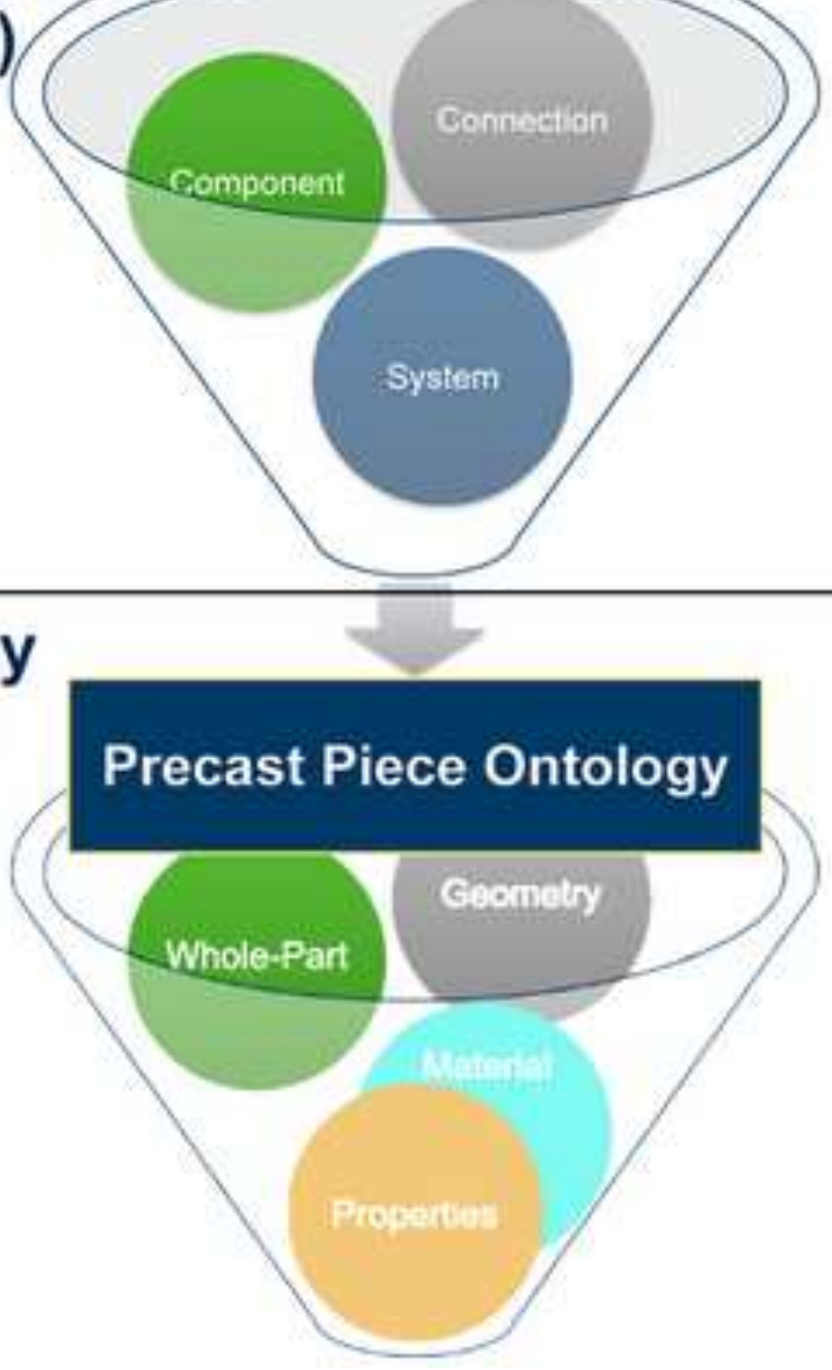

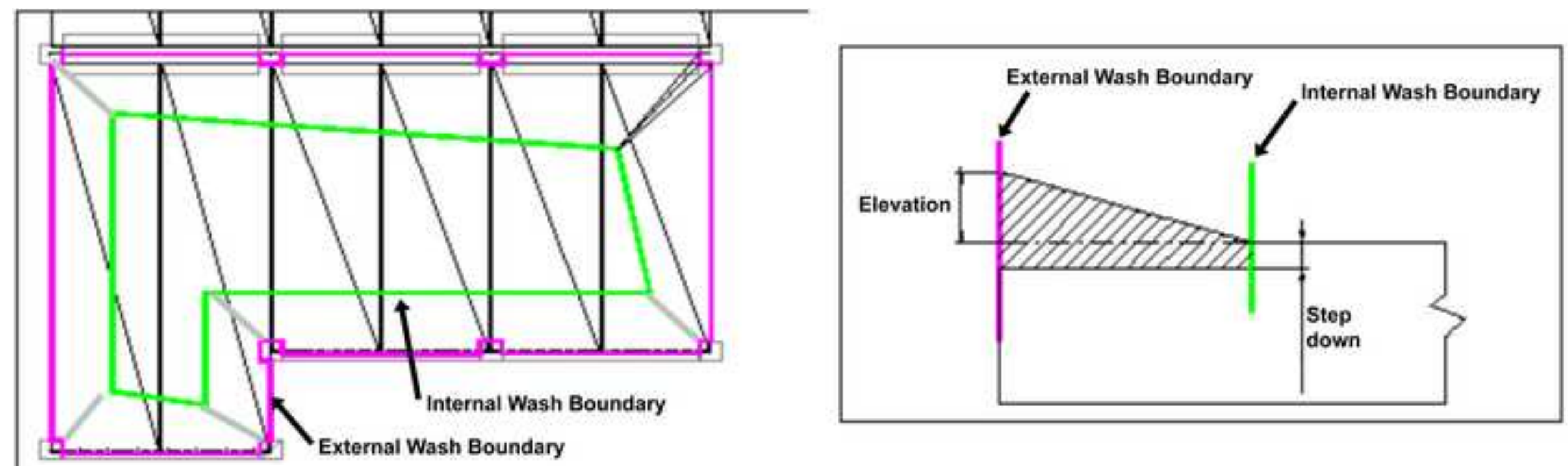


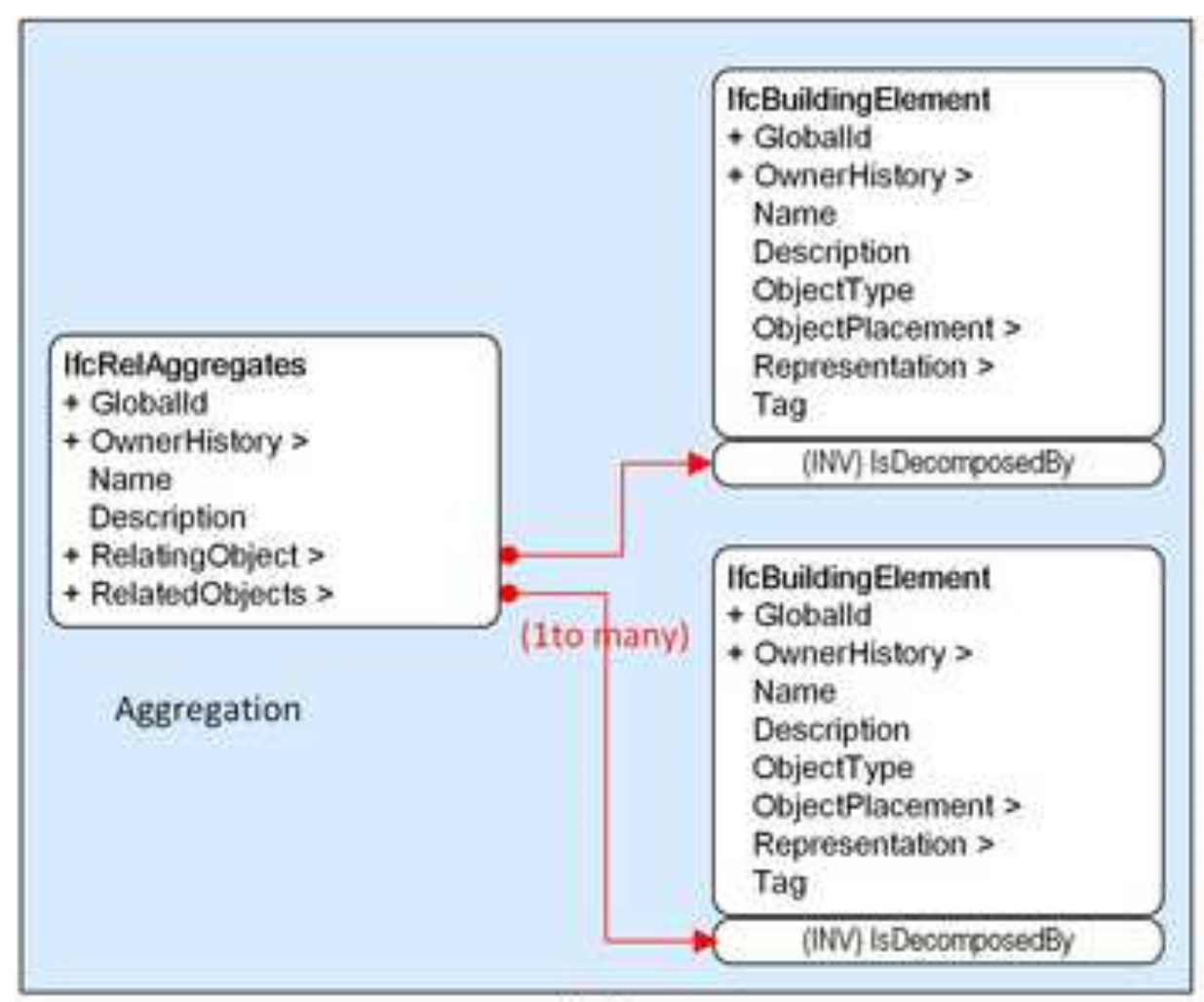

(a)

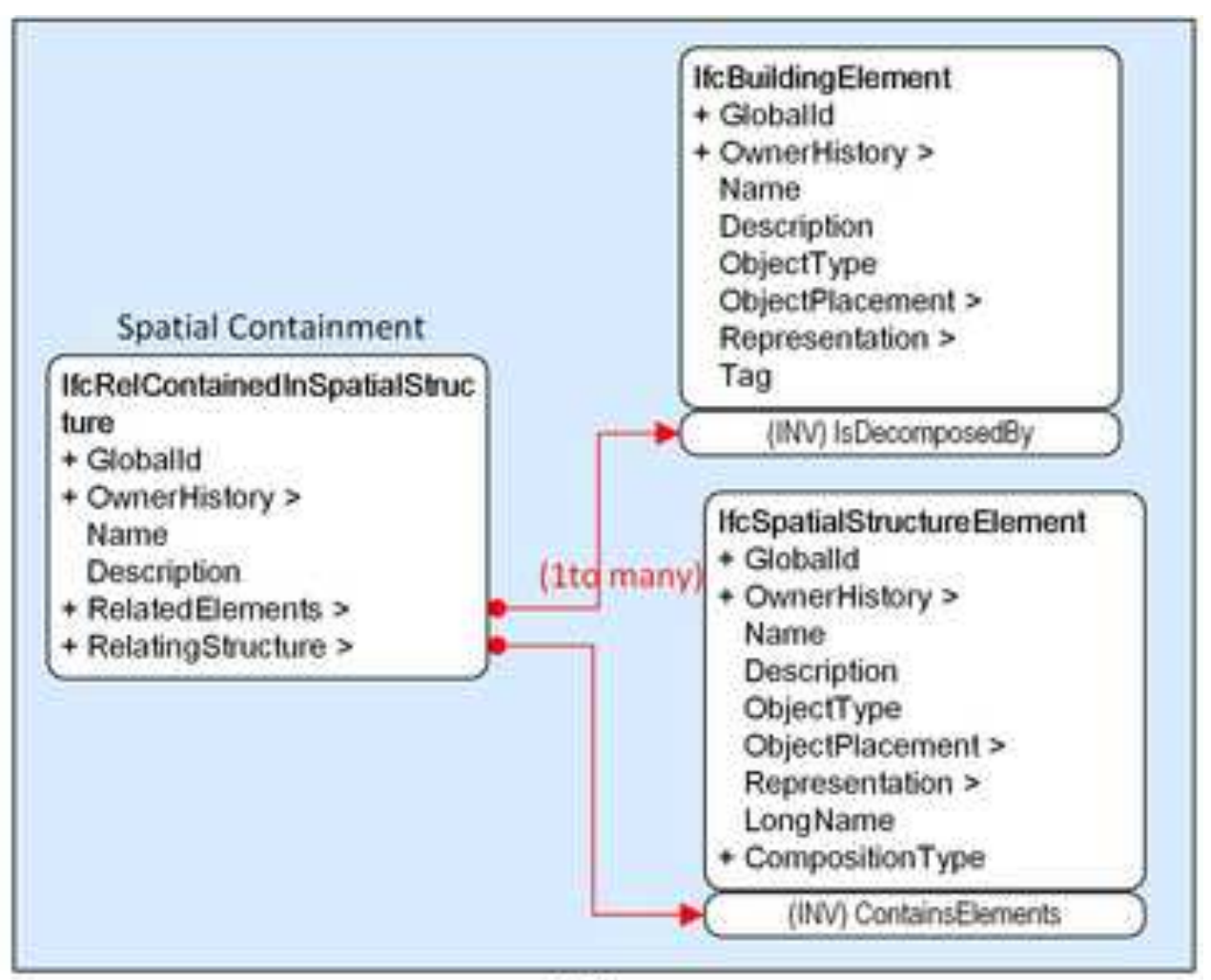

(b) 


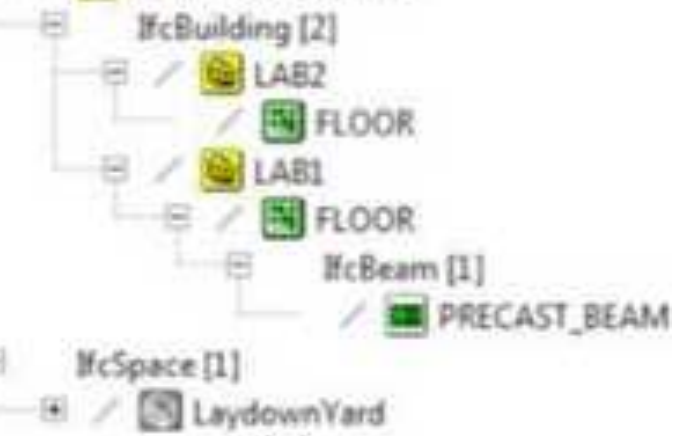

- QundownVard

(c)

Figure 09

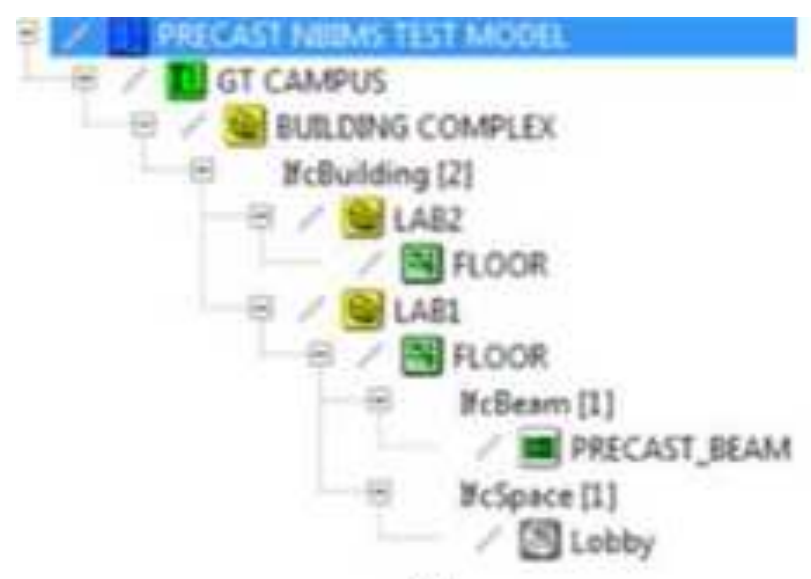

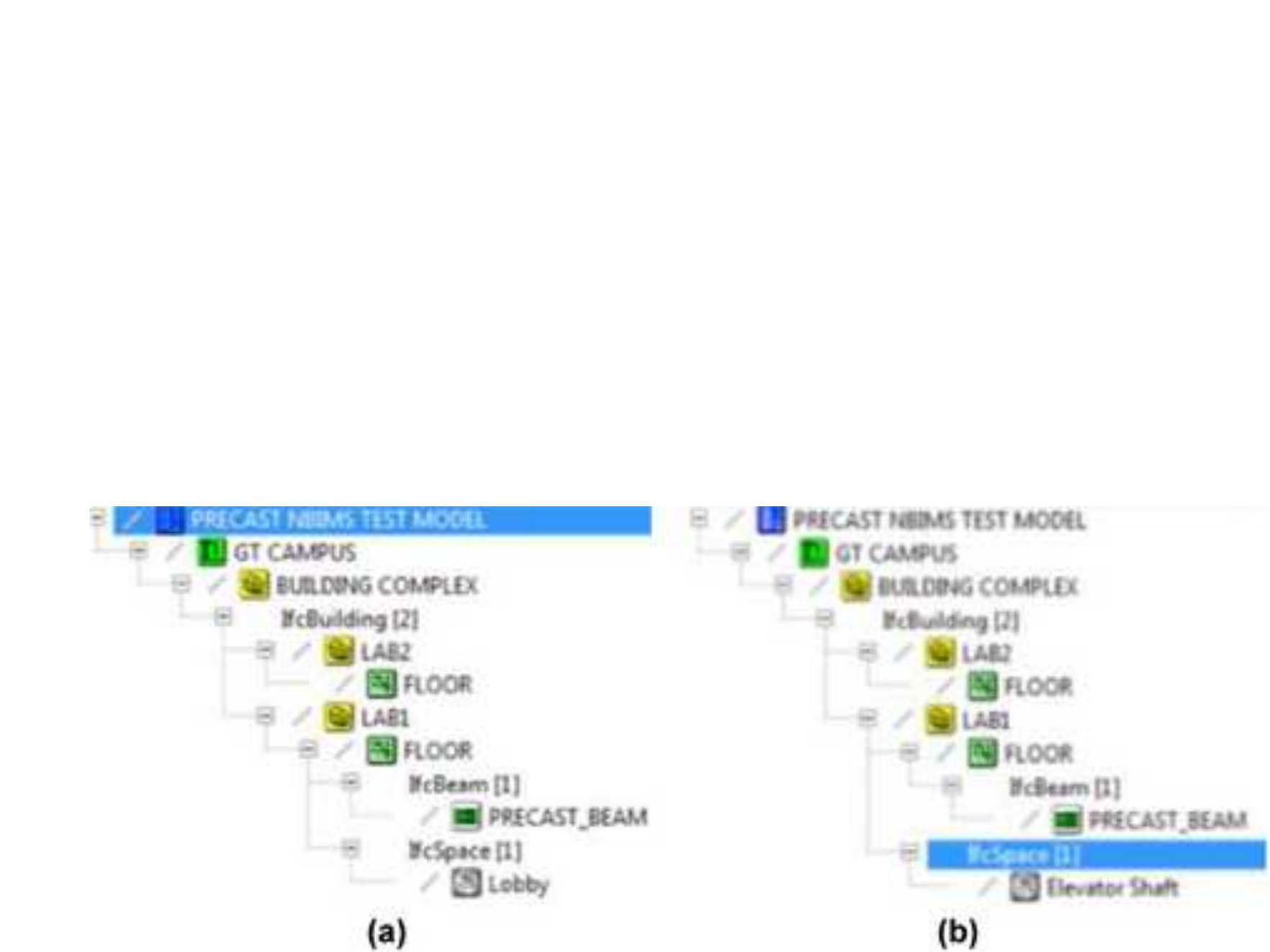

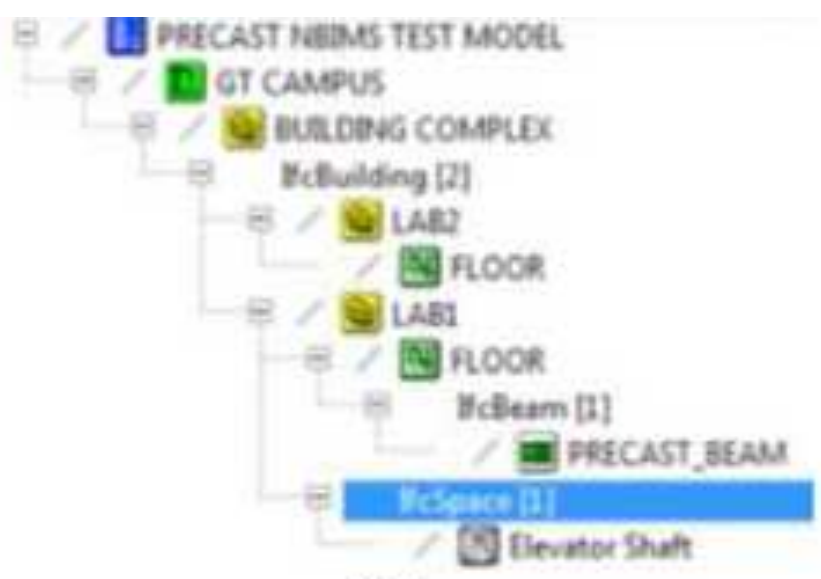

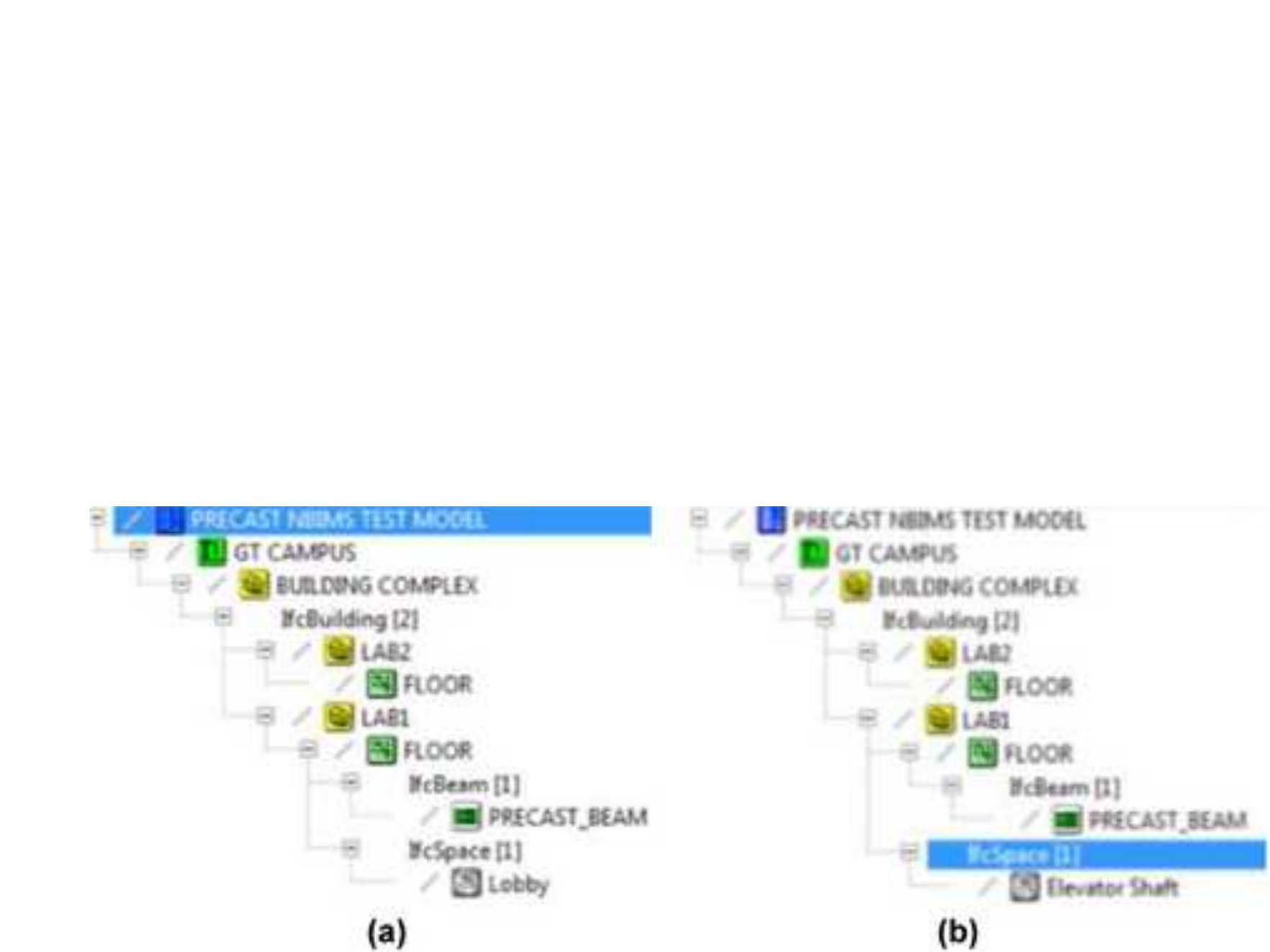




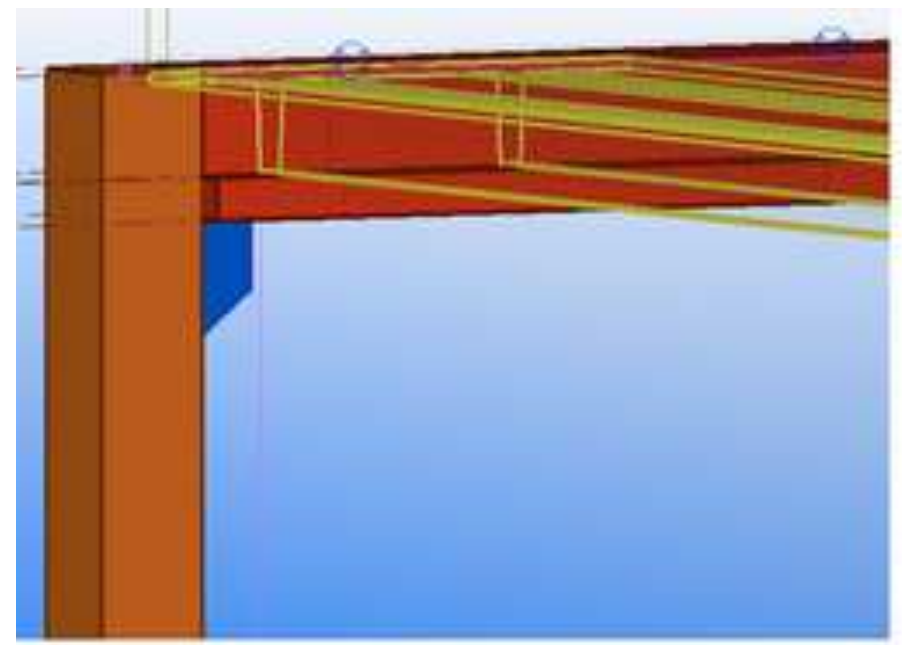

(a)

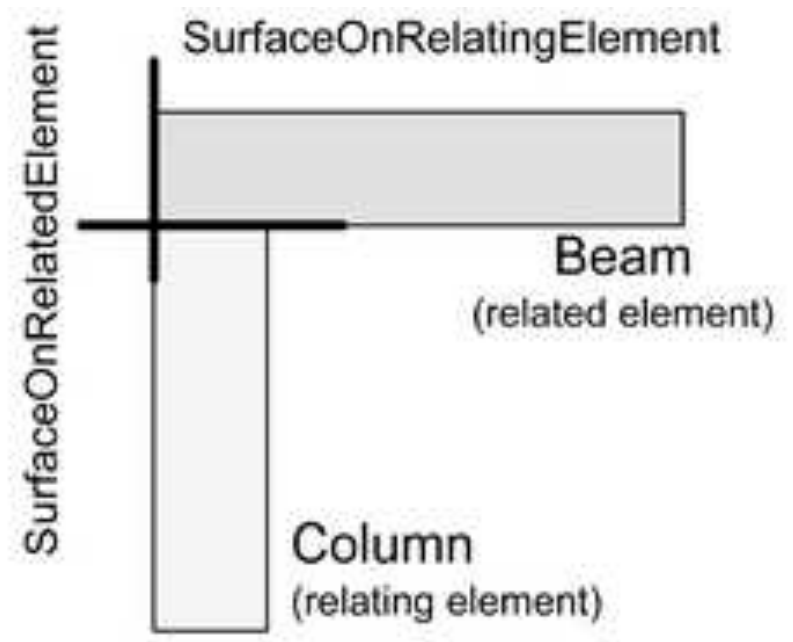

(b)

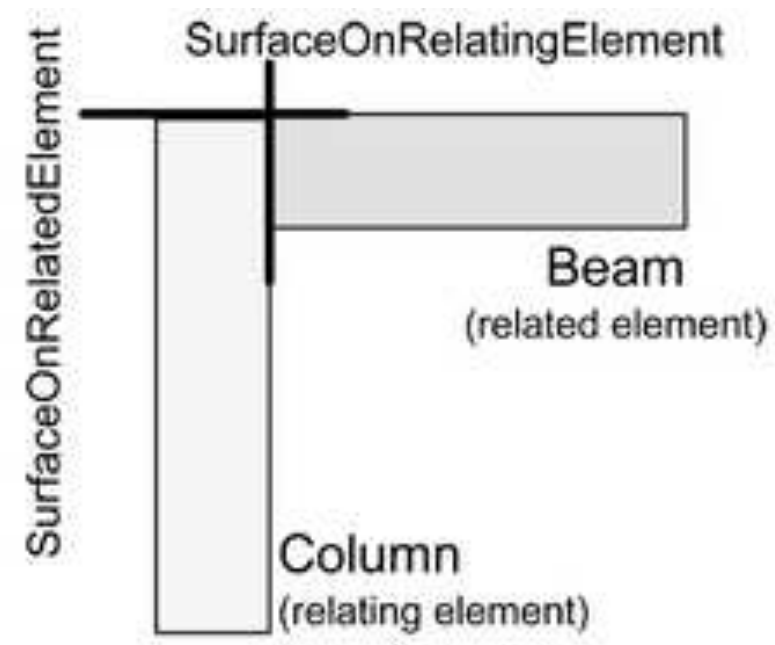

(c) 


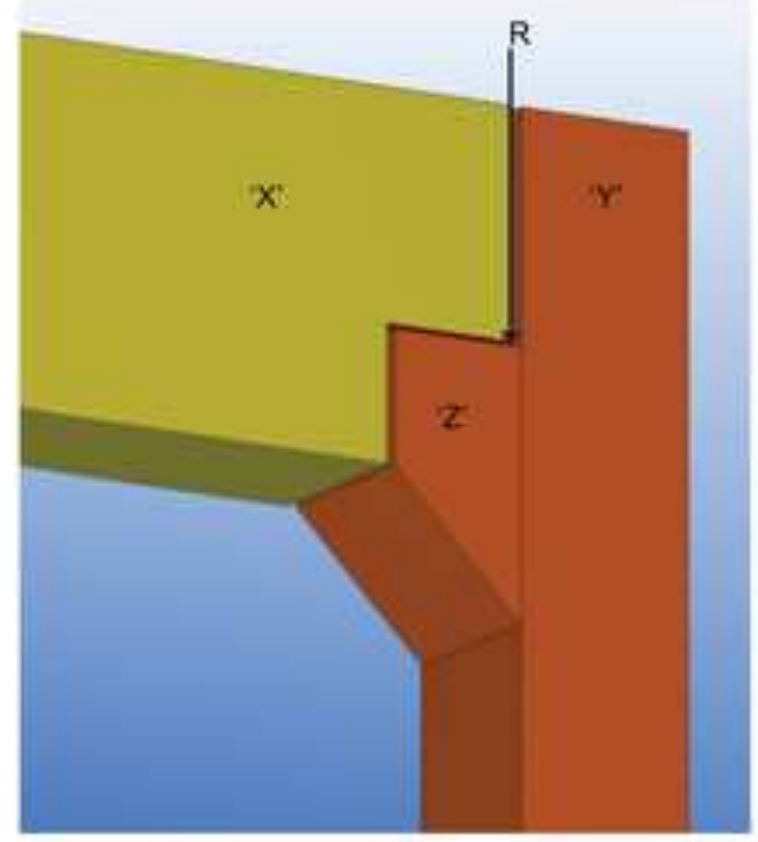

(a)

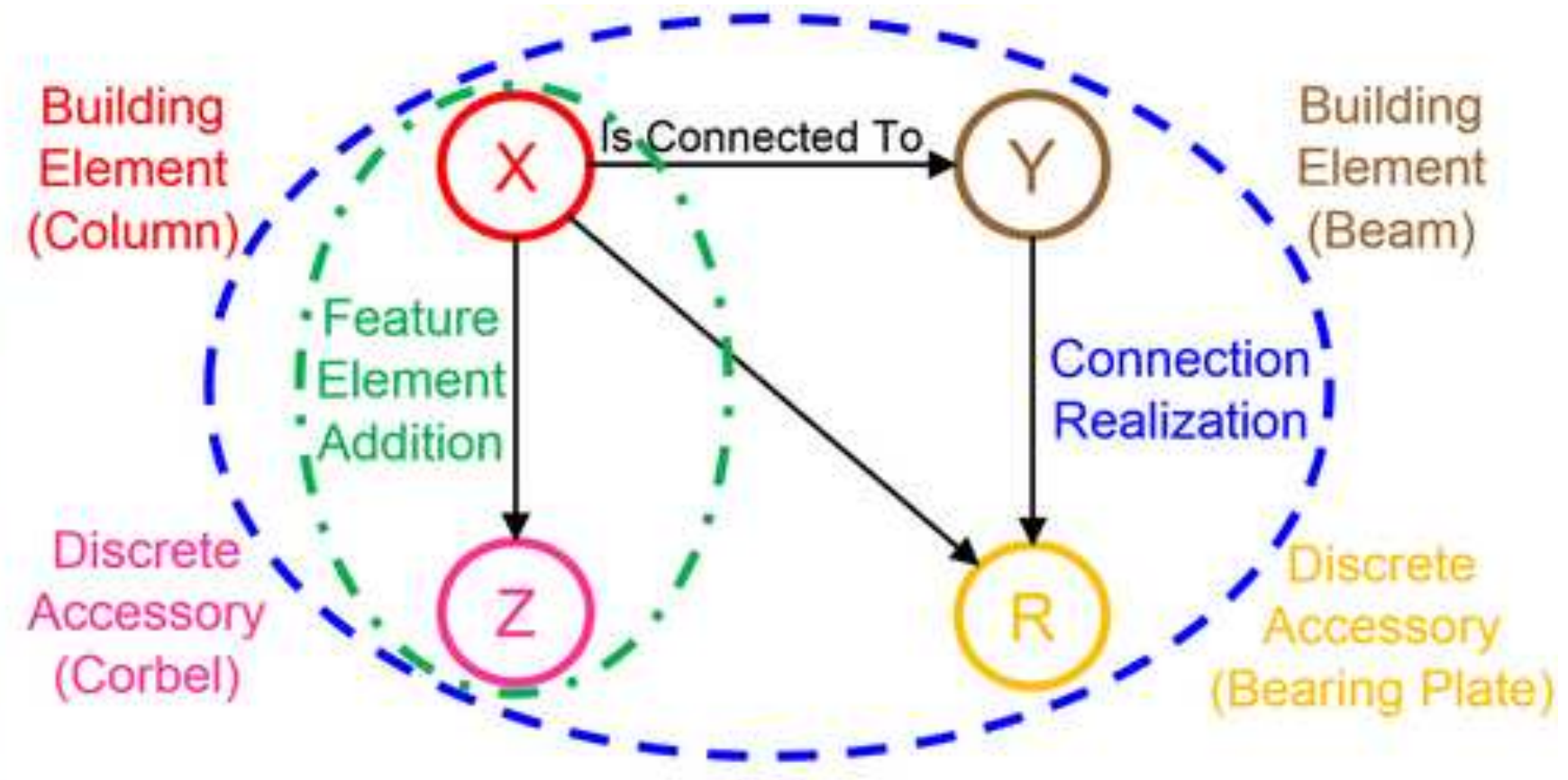

(b) 
Figure 12

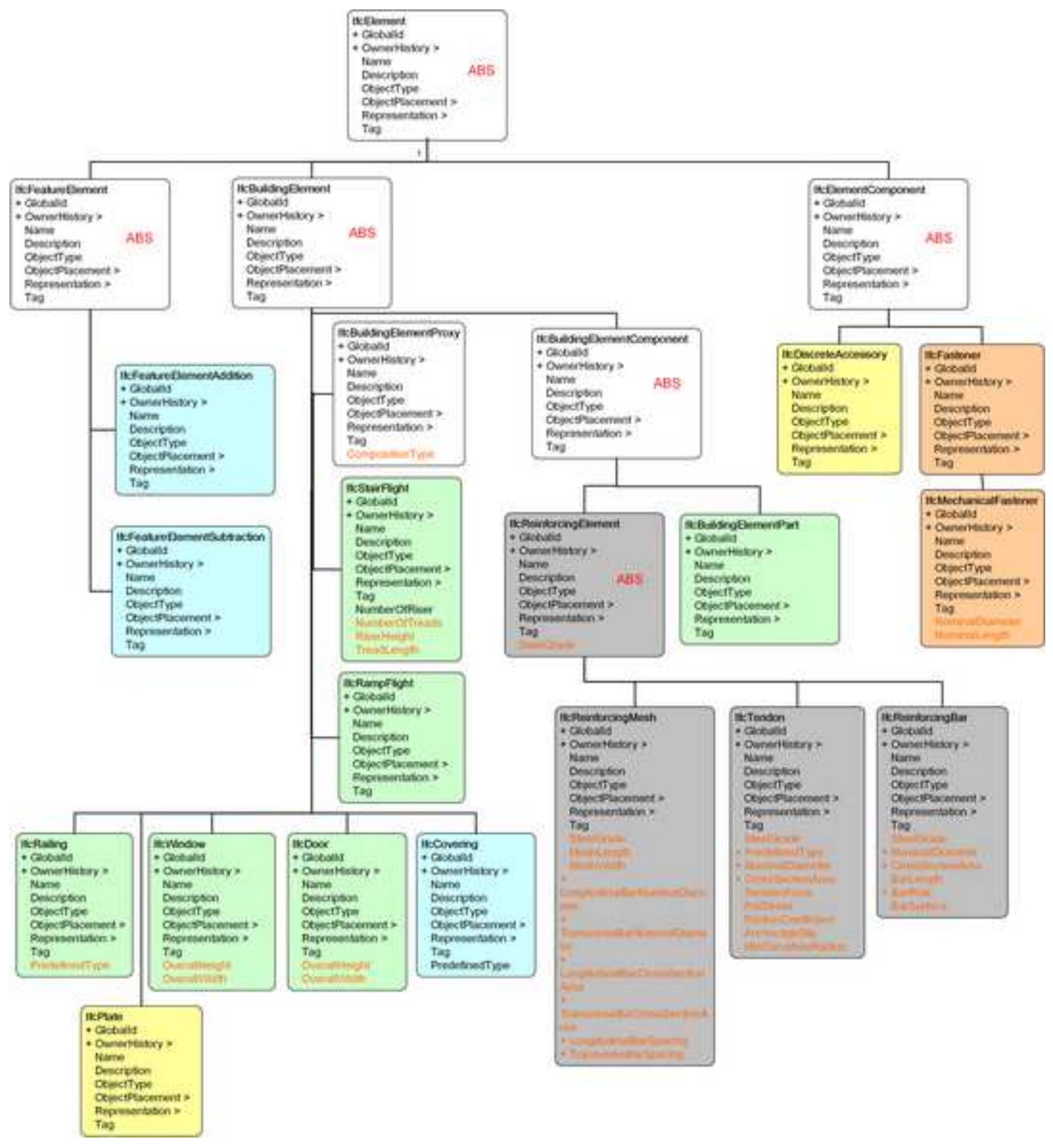

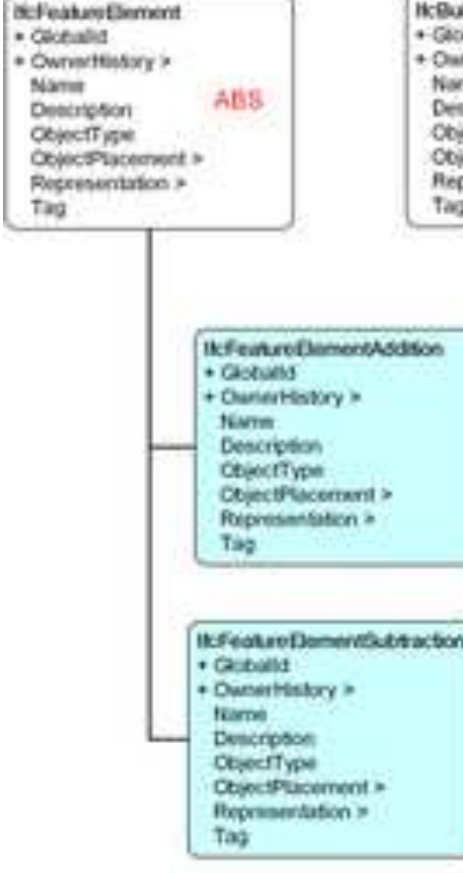

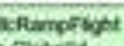
- Owneititiory. Name

Dencostion actoctros Copastacemaet? Peorisuntusion : Owbateser Nane

Dewryton

ctpertiks:

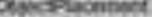

政 - Cacousid Nime

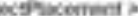
Amoresentulion?

tas

Muntersting

Simbestrition + Oenertilaver Oetiatia

absingenmenpy

Nare

orjectinceaver:

istembin

Iterandorang

opont

liame

Descrubon

Mepresectaten ?

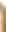




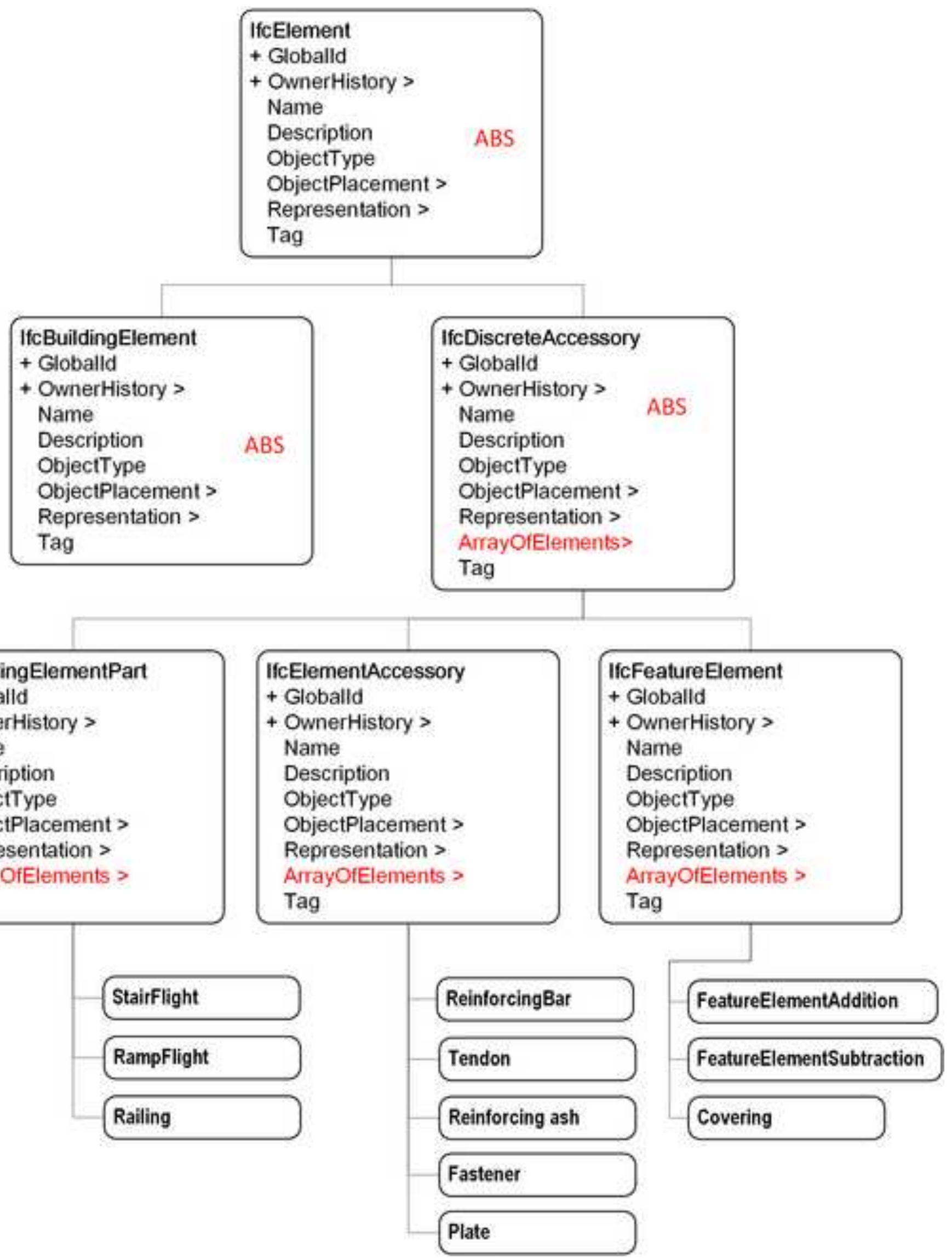

IfcBuildingElementPart
+ Globalld
+ OwnerHistory >
Name
Description
ObjectType
ObjectPlacement >
Representation >
ArrayOfElements >
Tag

\section{IfcBuildingElementPart}

+ OwnerHistory > ObjectType Representation > ArrayOfElements ?
IfcElement

OwnerHistory >

Name

Description

ObjectType

ObjectPlacement $>$

Representation >

Tag
OwnerHistory >

Name

Description

ObjectType

ObjectPlacement >

Representation >

ArrayOfElements>

Tag
+ OwnerHistory >

Name

Description

ObjectType

ObjectPlacement >

Representation >

ArrayOfElements >

Tag
ReinforcingBat

Tendon

Reinforcing ash

Fastener

Plate 